\title{
Promise Into Practice: Application of Computer Vision in Empirical Research on Social Distancing
}

\author{
Wim Bernasco ${ }^{1,2 *}$ ( ORCID 0000-0002-3385-0883 ) \\ Evelien Hoeben ${ }^{2}$ ( ORCID 0000-0003-0486-4175 ) \\ Dennis Koelma ${ }^{3}$ ( ORCID 0000-0002-2207-5682 ) \\ Lasse Suonperä Liebst ${ }^{2,4}$ ( ORCID 0000-0003-1062-2447 ) \\ Josephine Thomas ${ }^{2}$ ( ORCID 0000-0001-9835-1121) \\ Joska Appelman² ( ORCID 0000-0001-5311-3058) \\ Cees G. M. Snoek ${ }^{3}$ ( ORCID 0000-0001-9092-1556 ) \\ Marie Rosenkrantz Lindegaard ${ }^{2,4,5}$ ( ORCID 0000-0002-1630-774X )
}

\footnotetext{
${ }^{1}$ Department of Spatial Economics, School of Business and Economics, VU University Amsterdam, Amsterdam, The Netherlands

${ }^{2}$ Netherlands Institute for the Study of Crime and Law Enforcement (NSCR), Amsterdam, The Netherlands

${ }^{3}$ Video \& Image Sense Lab, Faculty of Science, University of Amsterdam, Amsterdam, The Netherlands

${ }^{4}$ Department of Sociology, University of Copenhagen, Copenhagen K, Denmark

${ }^{5}$ Department of Sociology, Faculty of Social and Behavioural Sciences, University of Amsterdam, Amsterdam, The Netherlands.

*Corresponding author. E-mail: wbernasco@nscr.nl

\section{Acknowledgements}

This work was supported by a grant from ZonMW (no. 10430022010017, Towards evidence-based social distancing policy). The authors thank Kiki Bijleveld at the NSCR for assistance in processing and coding video data, and Maikel van Scheppingen, Makki el Jouhri and Tineke Gortworst-Michels at the Amsterdam Police Department for assistance in securing video data.

\section{Author contributions}

Conceptualization - Overaching Idea: WB, MRL , LSL. Conceptualization - Article Narrative: WB, EH. Data Curation: JA, WB, DK, JT. Formal Analysis: WB, DK. Funding Acquisition: WB, EH, LSL, MRL (principal investigator), CS. Investigation: JA, WB, EH, DK, JT. Methodology: WB. Project Administration: JA, MRL. Resources: MRL, CS. Software: WB, DK. Supervision: WB, MRL, CS. Validation: JA, WB, DK. Visualization: WB, EH. Writing - Original Draft Preparation: WB, EH. Writing - Review \& Editing: JA, WB, EH, LSL, MRL, DK, CS, JT. 


\title{
Promise Into Practice: Application of Computer Vision in Empirical Research on Social Distancing
}

\begin{abstract}
Social scientists increasingly use video data, but large-scale analysis of its content is often constrained by scarce manual coding resources. Upscaling may be possible with the application of automated coding procedures, which are being developed in the field of computer vision. Here, we introduce computer vision to social scientists, review the state-of-the-art in relevant subfields, and provide a working example of how computer vision can be applied in empirical sociological work. Our application involves defining a ground truth by human coders, developing an algorithm for automated coding, testing the performance of the algorithm against the ground truth, and run the algorithm on a large-scale dataset of CCTV images. The working example concerns monitoring social distancing behavior in public space over more than a year of the COVID-19 pandemic. Finally, we discuss prospects for the use of computer vision in empirical social science research and address technical and ethical limitations.
\end{abstract}

\section{Keywords}

Computer vision, Video data analysis, Deep learning, Pedestrian detection, Social distancing

\section{Introduction}

Video data analysis promises to enrich the methodological toolbox of the social sciences (Nassauer and Legewie 2021), adding to traditional methods that offer a more coarse-grained picture of human behavior and interaction -including interviews (Jerolmack and Khan 2014, Small and Cook 2021) and on-site observations (Morrison, Lee, Gruenewald et al. 2016). On-site observation limits the data transparency and reproducibility of findings (Nassauer and Legewie 2021:141). In addition, some behavior is difficult, if not impossible, to record in a reliable manner when only having a single chance to observe it (Philpot, Liebst, Møller et al. 2019). This especially applies to interactions between multiple individuals. Further, retrospective interviews are not ideal to studying situational dynamics, in particular because respondents have imperfect memories and cannot always recall details of the situations they are interviewed about (Nassauer and Legewie 2021:141). 
What recorded video data brings to the stage is the ability to overcome the limitations of the human visual and cognitive system in processing high volumes of information at high speed. Recorded video data allows us to playback video data at any speed and conduct observations by as many observers as needed until measures are intersubjectively reliable.

A practical limitation is that watching, interpreting, and coding video data is time-intensive and errorprone for humans. With limited resources, large-scale analysis of video data is often not feasible. Automated coding of video data may provide a solution. Recent developments in computer vision have brought automated coding of video data within reach of the social sciences. Computer vision is wellsuited to contribute to the analysis of nonverbal and physical aspects of social life, including how social action manifests itself in behavioral displays (Goffman 1971), the co-present numbers and densities of the interacting participants (Turner 2010:57ff), and their organization in time and space (Kendon 1990:209ff).

Computer vision technology holds potential for upscaling the empirical analysis of video data because the efficiency of automated coding allows much larger and more generalizable samples than currently feasible (Lindegaard and Bernasco 2018:177, Nassauer and Legewie 2021:150-51). For example, the computer vision algorithm developed in the current study automatically coded a total of 66,175 still frames sampled from video recordings. Because the human coders used 2.2 minutes on average to code a single still frame, the algorithm's coding made up for 2,446 hours (i.e., 303 workdays of 8 hours) of human coding time.

Thus, computer vision adds volume and speed to the processing of video data, facilitating larger samples and thus opportunities to better quantify phenomena and determine generalizable relationships between them. The combination of video data and computer vision technologies may, therefore, solve the trade-off between the behavioral micro-details acquired by human video observation and the practical sample size restrictions that are inherent to this method.

The current paper aims to familiarize social scientists with computer vision and to inspire them to adopt it in their own work. In section 2, we outline what computer vision entails, which techniques have been developed to enable automatic human recognition, and for which type of research questions sociologists could use this method. In section 3, we offer a working example of the application of computer vision for studying social distancing in public space during the COVID-19 pandemic, and provide an overview of the steps necessary to develop an algorithm on a set of videos. In section 4, we elaborate on potential future applications of this algorithm, as well as on the challenges and limitations that social scientists should consider before adopting computer vision in their own work. 


\section{Computer vision in social science}

This section starts with a brief introduction to computer vision. It subsequently addresses the role that human assessment plays in evaluating the performance of computer algorithms and reviews the recent literature on two areas of research in computer vision: social signal processing and group and crowd analysis. Finally, we provide examples of studies that have addressed sociologically relevant questions with the aid of computer vision.

\subsection{Computer vision}

Computer vision is a field of scientific inquiry that aims to develop techniques for computers to automate tasks that the human visual system can do. These tasks include processing, analyzing, and understanding digital images, or sequences of digital images (videos). The objective is to make computers perform the visual tasks as good as humans, not necessarily to make computers do it in the same way humans do.

A digital image consists of a matrix of picture elements (pixels), each of which is characterized by a color or a shade of grey. Typically, these are photographs, and thus two-dimensional projections of the threedimensional world. Videos are sequences of images. When these are presented at a rate of more than 12 images per second, humans can no longer distinguish the individual images in the sequence. This gives the impression of an uninterrupted stream of visual information, possibly including smooth movement. Image classification and object detection are the most common applications of computer vision. Image classification is the task of determining, from a set of predefined alternatives, which label best describes an image (e.g., whether the image shows a street light or a traffic light). Object detection is the task of determining which objects are visible in an image (e.g., a traffic light, a motorcycle, and a human being), and marking wherein the image they are located (e.g., by defining lines around their boundaries).

Most of the visual tasks in computer vision are easy for humans but have proven very difficult for computers. An everyday example is the CAPTCHA ${ }^{1}$, a visual puzzle that some websites use to identify the user as a human. Some of these puzzles require the user to locate common objects like traffic lights or motorcycles in an image. Most humans solve these visual puzzles in seconds, but computers do not. However, the promise of computer vision is that once computers do become successful at a task, they can perform it at great speed and low cost.

Computer vision is a subfield a machine learning, a method of analysis that automates model building with very little human intervention or instruction. It is common to distinguish between supervised

\footnotetext{
${ }^{1}$ CAPTCHA is a contrived acronym for "Completely Automated Public Turing test to tell Computers and Humans Apart".
} 
learning (where the computer learns from examples constructed by humans) and unsupervised learning (in which the computer automatically creates clusters in data or reduces the dimensionality of the data).

The main computational technique that has spurred recent development in computer vision is deep learning (LeCun, Bengio and Hinton 2015). It is a class of algorithms that rely on computing systems called neural networks to detect patterns in data. When a neural network is provided with a large number of example images that have been classified by humans, it is able to determine common patterns in those examples and to use this 'knowledge' to independently classify future unclassified images. ${ }^{2}$

Examples of practical areas where computer vision is applied are medical diagnosis (e.g., detecting malignant tumors, see Shia and Chen 2021), surveillance (e.g., by face recognition, see Harikrishnan, Sudarsan, Sadashiv et al. 2019), and self-driving cars (e.g., detecting the presence of pedestrians in traffic situations, see Hasan, Liao, Li et al. 2020). Our example application in the present paper uses a customized computer vision algorithm for pedestrian detection.

\subsection{Human annotation}

To evaluate and improve algorithms, we need feedback on how well they perform their tasks. Deep learning techniques in particular need large numbers of annotated images to train their neural networks in all stages of development, including the initial training of the model. Feedback requires a ground truth to serve as a performance benchmark. To know whether a computer performs this task correctly, a human coder — performing the same task as the algorithm— provides the ground truth. The act of a human viewing and coding images is called annotation. Without annotated images there is no way to evaluate the performance of computer vision algorithms. Because human annotators can make errors, interrater reliability assessment of human annotation is required to verify that annotation error margins are small.

An annotation tool is a piece of software that allows human coders to identify and mark locations and properties of the image objects that need to be detected and classified. Because different tasks require different kinds of annotations, there are many different annotation tools tailored to specific tasks. Some annotation tools are freely available online (Dutta and Zisserman 2019, Forsyth and Ponce 2012: 513517).

Annotation tools typically provide four main functions. First, they present the visual material to be coded to the human annotator, one at a time. This may include options like zooming in, and moving the viewing window across the image horizontally or vertically. For video clips, it may also include options for

\footnotetext{
${ }^{2}$ Textbook introductions are Szeliski (2011) and Forsyth and Ponce (2012) on computer vision in general, and Goodfellow, Bengio and Courville (2016) on deep learning. Torres and Cantú (2021) provide an introduction of the use of computer vision in social science research.
} 
rewinding, repeating, and setting the speed (i.e., slow-motion). Second, they allow the human annotator to mark the location of objects. Locations of objects are typically identified using bounding boxes. A bounding box is the smallest rectangle that completely includes the detected object. Third, labels or 'tags' can be attached to identified objects to record their observed properties. For example, if the task is to read vehicle number plates, the tag would contain the number on the plate. Finally, the annotations are stored so that they can be used to evaluate the performance of the computer algorithm.

Computer vision scientists and practitioners train and evaluate their algorithms on benchmark datasets of images annotated by humans. Many benchmark datasets relevant for detecting persons have recently been created in the context of software development for autonomous driving, where quick and reliable automated detection of pedestrians and other traffic participants is crucial to prevent collisions (Cao, Pang, Xie et al. 2021, Hasan et al. 2020). Benchmark datasets typically contain the original images and their annotations: the validated characteristics of successfully detected objects, including at least their bounding boxes and possibly also additional attributes.

\subsection{Performance evaluation}

As explained in the previous section, to assess the performance of a computer vision algorithm, its outcomes are quantitatively compared to the ground truth as defined by human coders in annotated datasets. Depending on the vision task at hand, various performance measures can be used. For binary classification (e.g., to decide whether there is a person visible anywhere in an image), performance can be measured with simple statistics like precision and recall. If the task is more complex, such as in object detection (e.g., to find in an image all persons and their locations), the performance assessment will have to weigh the amount of inaccuracy in localization as well. Common performance assessment statistics in these situations include the log-average miss rate, average precision, and the Jaccard index (Cao et al., 2020).

\subsection{Advances in human behavior recognition}

Within the rapidly developing field of computer vision, we distinguish two research lines that hold particular promise for social science research: social signal processing (Burgoon, Magnenat-Thalmann, Pantic et al. 2017, Vinciarelli, Pantic and Bourlard 2009) and the recognition of persons, groups, and crowds (Murino, Cristani, Shah et al. 2017b, Zitouni, Sluzek and Bhaskar 2019).

\subsubsection{Social signal processing}

Social signal processing concerns the use of computer vision techniques for the extraction of emotional and behavioral cues in individuals, dyads, and small groups based on the analysis of gestures, body posture, gaze direction, and facial expressions. This research generally applies video recordings from eye- 
level cameras collected in controlled environments. Thus, it relies on recordings with good illumination, relatively stable camera angles and zoom-levels, subjects in frontal view, few occlusions (no objects or other subjects blocking the view), and audio recordings accompanying the visual information.

Many studies of social signal processing focus on automatic emotion recognition from facial expressions (for overviews, see Canedo and Neves 2019, Martinez 2019) or body postures (for overviews, see Kleinsmith and Bianchi-Berthouze 2013, Stephens-Fripp, Naghdy, Stirling et al. 2017). Advancements have been made to distinguish between spontaneous and posed (deliberately produced) behaviors (Bartlett, Littlewort, Frank et al. 2014, Valstar, Gunes and Pantic 2007, Yang, Hossain, Gedeon et al. 2020) and to detect deception (Bruer, Zanette, Ding et al. 2020, Tran, Sen, Haut et al. 2020, Zanette, Gao, Brunet et al. 2016).

Although the majority of the studies examined individual-based emotion detection, the emotion analysis of groups has gained interest in recent years (see overview by Veltmeijer, Gerritsen and Hindriks 2021). Other efforts to assess social relationships have focused on interactional traits such as warmth and dominance (Black, Katsamanis, Baucom et al. 2013, Hung, Jayagopi, Yeo et al. 2007, Jayagopi, Hung, Yeo et al. 2009, Sanchez-Cortes, Aran, Mast et al. 2012, Zhang, Luo, Loy et al. 2018) or on role categorizations such as the distinction between kinship, friendship, romantic, and professional relationships on still images (Li, Wong, Zhao et al. 2020, Sun, Schiele and Fritz 2017) and on video clips (Lv, Liu, Zhou et al. 2018).

Finally, projects have started to enable the (semi-)automatic measurement of social cohesion within small groups based on non-verbal social cues, such as gaze, head nods, laughter and turn taking (Hung and Gatica-Perez 2010, Kantharaju, Langlet, Barange et al. 2020, Walocha, Maman, Chetouani et al. 2020).

\subsubsection{Recognition of persons, groups, and crowds}

In computer vision, 'group and crowd analysis' concerns the detection of persons and groups within crowds, and of the tracking of the velocity, direction, and other movements of persons, groups, and crowds. This research line uses real-life video recordings from cameras at elevated viewpoints, such as surveillance cameras attached to upper levels of buildings or to unmanned aerial vehicles (i.e., drones) and satellite cameras.

Within this line of work, the detection and tracking of persons still represent one of the core challenges (Hasan et al. 2020, Murino, Cristani, Shah et al. 2017a). A related challenge is pose estimation, which involves locating the individual joints of the human body (right elbow, left wrist, etc.) in images containing multiple persons (Cao, Hidalgo, Simon et al. 2019). At the same time, the field has started to explore new venues of research such as the identification of small groups within crowds (Vascon and 
Bazzani 2017) based on the proximity between individuals, similarities in trajectory patterns (Ge, Collins and Ruback 2012, Hu, Zhang, Song et al. 2021), mutual poses (Tran, Bedagkar-Gala, Kakadiaris et al. 2013) and the various formations groups can take, as indicated by members' head and body orientation. For example, while moving in a crowd, group members can walk side by side or in a V-shaped pattern, depending on the density in the crowd (Moussaïd, Perozo, Garnier et al. 2010). Groups that stand still in conversation can display side-by-side, face-to-face, L-shaped, and circular formations (Setti, Russell, Bassetti et al. 2015).

Building on this knowledge, scholars have also been able to automatically distinguish leaders from followers within such small groups, based on their physical centrality and their influence on the physical dynamics within the groups (Solera, Calderara and Cucchiara 2017, Yu, Lim, Patwardhan et al. 2009). At the crowd level (for overviews, see Grant and Flynn 2017, Varghese and Thampi 2018), researchers have worked at ways to automatically assess, for example, the density of the crowd (Almagbile 2019, Rodriguez, Sivic and Laptev 2017), the level of excitement in the crowd (Baig, Barakova, Marcenaro et al. 2014, Conigliaro, Rota, Setti et al. 2015, Varghese and Thampi 2018), and the presence of anomalies in the crowd (for overviews, see Nayak, Pati and Das 2021, Sodemann, Ross and Borghetti 2012, Tripathi, Jalal and Agrawal 2018). Removing crowd size limitations of prior methods, Cruz and González-Villa (2021) proposed a method to estimate crowd sizes on high-resolution images from gigapixel cameras, in which images typically contain many thousands of individuals.

Two related areas of inquiry are activity recognition, in which human actions are automatically recognized and classified (for literature reviews, see Herath, Harandi and Porikli 2017, Sigurdsson, Russakovsky and Gupta 2017) and event recognition, which aims at recognizing and classifying events, including social events (for a literature review, see Tzelepis, Ma, Mezaris et al. 2016). Based on sudden temporal and spatial motion changes, it is possible to detect instances of violence (Chang, Krahnstoever, Lim et al. 2010, Mohammadi, Galoogahi, Perina et al. 2017, Roman and Chávez 2020, Tripathi et al. 2018), panic-driven events (Haque and Murshed 2010), and snatch theft (Roy and Mohan C 2018) and various other specific criminal actions (Sultani, Chen and Shah 2018).

\subsection{Promises for sociology}

Despite their potential, few scholars have applied computer vision techniques to theory-driven sociological research. Most studies appear to focus on practical applications rather than on the advancement of sociological theory. Without sketching pipe dreams, we discuss five potential research areas that could be addressed with the aid of currently available computer vision techniques. 
First, computer vision enables the large-scale examination of the link between social structural categories (e.g., assumed gender, age, ethnicity) and behavior in public space. For example, based on video footage Stipancic, Zangenehpour, Miranda-Moreno et al. (2016) found that male-presenting cyclists were less likely than female-presenting cyclists to be involved in near-crash events with vehicles. Zhang, Chen and Li (2020) analyzed the contents of geo-tagged social media photos and found differences between Asian, American, and European tourists in their preference for architecture, natural scenes, plants, food, and entertainment. Dietrich and Sands (under review) analyzed traffic camera footage of pedestrian behavior to examine inter-group racial avoidance. Obviously, the hazards of non-inclusive categorization as well as possible errors in assigning category-membership that plague human observers, also applies to algorithms.

Second, computer vision could aid in the identification of groups and their informal leaders based on recorded informal behavior. For example, recordings from a high school canteen during lunch break or a prison courtyard could be used for this purpose (Yu et al. 2009). Sociologists currently rely heavily on questionnaires to obtain sociometric information (for recent meta-analyses on social network studies, see Gallupe, McLevey and Brown 2019, Ivaniushina and Titkova 2021), and computer vision might provide a useful addition (or even alternative) to this method.

Third, computer vision techniques could be used to characterize outdoor behavior patterns in neighborhood research. Such techniques have already been used in neighborhood research to map land use and other physical neighborhood conditions, often relying on Google Street View or satellite imagery (Khorsidi et al., 2021; Naik et al., 2017). Such work could be expanded with observations of objects (such as cars, Gebru, Krause, Wang et al. 2017) and pedestrians at those locations, which could add information about demographics and socioeconomic status. Also, advancements are being made in 'emotional computing,' which uses facial expression-based emotion detection on geo-tagged user-generated content to assess trends in emotions at specific locations (Huang, Fei, Kwan et al. 2020, Liu, Yuan and Zhang 2020). This is promising for research on the link between people's surroundings and their feelings of safety or well-being.

Fourth, the automatic detection of nonverbal behavior in dyadic or group interactions has been applied in experimental set-ups with fixed cameras to distinguish liars from non-liars among children (Bruer et al. 2020) and to predict the success of learning in student-teacher dyadic interactions (Won, Bailenson and Janssen 2014), the outcome of salary negotiations (Li, Curhan and Hoque 2018), and the effectiveness of social skills training (Chen, Chang, Tian et al. 2015). 
Fifth and finally, the automatic detection of anomalies within crowd behavior could aid sociological research by identifying rare but interesting events from large amounts of video footage, to select for further (manual) examination. This would allow researchers to seek out footage of, for example, violent events, panic-driven behavior, and crime (Sultani et al. 2018).

To put the promises of computer vision for sociological research into practice, it is important to provide concrete examples of how computer vision methods can be used to address sociological research problems, and in particular which steps are needed to upscale data collection and analysis while maintaining appropriate controls on data quality. Our contribution provides such a working example.

\section{Working example on social distancing}

The present section describes the development and application of a computer vision algorithm. The purpose of the algorithm is to help describe public compliance with social distancing directives over the course of the COVID-19 pandemic. After introducing the substantive research question, we describe how ground truth was established with the help of human coders, how the algorithm was developed and applied, and how the results were used to plot trends in social distancing over time.

\subsection{Research problem}

Social distancing has been a critical measure to mitigate the spread of viral infections, including the COVID-19 virus (World Health Organization 2009). Across the globe, authorities have urged citizens to adapt their lifestyles and to keep a physical distance from other people. Although fear of infection may offer a strong incentive to comply with such directives, compliance is still difficult to maintain because it requires constant awareness and coordination and because it goes against common rituals in everyday practices in many societies (Hall 1966:131-63, Sorokowska, Sorokowski, Hilpert et al. 2017).

Whether people are willing and able to adapt their spatial and social behavior to new situations to support a collective good, and which factors affect the extent to which they do, are important questions of broader significance than the current pandemic (Collins 2020). For example, researchers have linked such compliance to cultural differences in strictness of adherence to social norms, finding that 'cultural tightness' can be an evolutionary advantage because it helps groups to cooperate faster when under threat (Gelfand et al., 2021). Social distancing behavior also reflects people's attitudes towards others and can, therefore, be used to assess interpersonal relationships (Hall, 1966: 113-130) and responses to out-group members (Bogardus 1933, Dietrich and Sands 2021).

From a practical standpoint, authorities need information on social distancing compliance to inform their policy decisions because the effectiveness of social distancing directives in reducing the spread of viruses 
depends on the cooperation of the public (Bauch and Galvani 2013). There is, however, little reliable systematic evidence on whether people keep a distance from others during epidemics.

In the current study, we develop and test an algorithm for automatically detecting the presence of humans and their compliance with social distancing rules from CCTV video recordings. We focus on the extent to which people keep a 1.5-meter distance from others in public space. The 1.5-meter threshold is advised in The Netherlands, where our study takes place. The few studies that have also studied social distancing behavior using video data (Pouw, Toschi, van Schadewijk et al. 2020, Rahim, Maqbool and Rana 2021, Zuo, Gao, Kurkcu et al. 2021) focus primarily on the required computer science techniques, and do not speak to a social science audience. The aim of the present study was to measure and monitor, over the course of the pandemic, the level of compliance to social distancing directives in urban public space. In doing so, we aim to contribute to the literature on social distancing (Hall, 1966) as well as to the public health literature on non-pharmaceutical measures in epidemics (Fong, Gao, Wong et al. 2020, World Health Organization 2009).

\subsection{Process overview}

Because computer vision has only recently emerged in social science research, there are usually no outof-the-box computer vision algorithms available that can be applied immediately to help answer substantive research questions. Instead, the algorithms must be developed from scratch, or existing algorithms must be adapted. Even if succesful algorithms are available, they have been trained on specific datasets, and would still need to be validated on a sample of the empirical data that inform the research question at hand.

In our application of computer vision for measuring social distancing compliance, we distinguished three stages. These stages are summarized in Figure 1. Each stage consists of a series of smaller steps that are described in the following sections.

\section{INSERT FIGURE 1}

\subsection{Determine ground truth}

To assess whether a computer vision algorithm does what it is supposed to do, we need a ground truth that functions as a benchmark. Because the algorithm is to replace a human observation task, human observation is the benchmark against which we evaluate the performance of algorithms.

\subsubsection{Collection of video data}

Data for the development of the algorithm were sampled from 1,059 recordings, captured by 53 municipal surveillance cameras in Amsterdam, the Netherlands. These recordings were collected over the course of 
7 weeks during the COVID-19 outbreak, from April 9 to May 24, 2020. This period was chosen because the corresponding video recordings were available at the time. The recordings are provided by the municipality of Amsterdam (the owner of the recordings), and were collected with the help off the Amsterdam Police Department (where the recordings are processed). The surveillance cameras are located in areas where the municipality deems supervision necessary. They are relatively crowded areas, both in the city center and in more peripheral neighborhoods, characterized by concentrations of retail businesses and public transportation hubs. Recordings were available between 9 AM and 8 PM on Thursdays, Saturdays, and Sundays. Securing, storing and converting a complete seven-day coverage would require more human and computer resources than the police could offer. Further, based on prior work on time use (e.g., Wikström, Oberwittler, Treiber et al. 2012), the three days were selected under the expectation that both days of the weekend and a weekday would allow a representative sample.

The video surveillance system stores recordings in a proprietary format (G64X) to prevent image tampering. To allow further processing, all recordings were first converted to the more common nonproprietary MP4 format using a tool (Genetec Player) provided by Genetec. ${ }^{3}$

Access to the video recordings was provided under the condition that the data would be securely stored, viewed and analyzed within offline lab facilities situated at a scientific institution, not be publicly shared, and that the identity of the persons visible on the recordings would be protected at any time. The project has been approved by the Ministry of Justice (PaG/BJZ/49986). The following supplemental materials have been made available at osf.io/yw67g: (1) the source code of the annotation tool (Python code), (2) all human-coded and algorithm-coded still frame data, but not the still frames themselves, and (3) the source code of the data analysis ( $\mathrm{R}$ code).

For both the determination of ground truth and the algorithm performance evaluation, we randomly sampled 60 recordings from the total collection of 1059 recordings. As the purpose of the research did not require that we measured the presence of people and social distancing per second or per minute, we sampled and extracted still frames from the 60 video recordings at full hours between 9 AM and 8 PM. The sample size of 60 was based on the expectation that it would yield 500-600 still frames. Because it was estimated that 100 still frames would be sufficient to determine inter-coder reliability, this sample of 60 recordings would allow for at least four iterations of improving inter-coder reliability should that be necessary.

For transparency, and to acknowledge and emphasize that trial and error were involved in setting up the research, we note that in an initial setup that was later discarded, we (1) used a single coder and thus did

\footnotetext{
${ }^{3}$ See www.genetec.com.
} 
not code inter-coder reliability, (2) included an additional measure of 'group formation' in addition to 'social distancing violation', (3) instructed the human coder and the algorithm to count numbers of people, social distancing violations and group formation during a 5 minutes every full hour, and (4) did not use an annotation tool (coders and algorithm exported numbers into a spreadsheet). The difficulty of defining clear and workable observation rules for the coder and the simultaneous realization that interrater reliability was to be tested, as well as disappointingly low outcomes of the performance tests, made us redesign the procedures.

Because due to technical issues not all recordings contained information about all 12 hours between 9AM and $8 \mathrm{PM}$, the sample of 60 recordings included 550 still frames in total. A practical advantage of sampling still frames from video recordings is that the coding of still frames is much less time-consuming than the coding of a video stream (an argument that is obviously more salient for human coding than for automated coding). Table 1 shows the subdivision into three subsets. As explained below, the purpose of the subdivision is to separate data used for training from data used for testing.

\section{INSERT TABLE 1}

\subsubsection{Annotation tool}

To facilitate and safeguard the reproducibility of the human coding process, an annotation tool was developed. The tool presented the human coder with a still frame on the screen. The coder was instructed to indicate each human being observed on the screen by marking their head with a red dot. Further, if the coder observed two or more people who were within 1.5-meter proximity of each other, they were marked with a yellow line between the red dots. After marking all visible human beings and all 1.5-meter distance violations, the coder moved on to the next still frame and repeated the process. The marked people, the distance links between them, and their location on the screen were automatically saved for each still frame. An example screen is presented in Figure 2.

\section{INSERT FIGURE 2}

\subsubsection{Outcome measures}

The visual task of both the human coders and the computer vision algorithm was to detect all instances of humans, to locate each of them in the image and to assess whether pairs of humans were at less than 1.5meter from each other. We used two simple outcome measures to assess performance, both for the human coding and the algorithm. They are the number of people present and the number of social distancing violations. Using only counts and ignoring locations does not mean that recording locations of persons and violations is redundant. During training and development, recorded locations can help to detect the 
sources of any potential discrepancies amongst human coders or between human codings and automated codings.

\subsubsection{Training human coders}

To determine a ground truth, two human coders were trained to independently assess the still frames with the annotation tool. Both coders received a 3-day training in which they were instructed on how to use the annotation tool for identifying and marking people and 1.5-meter distance violations. During this training, the human coders first practiced the coding of several frames together. Then, they coded several frames independently and extensively discussed the discrepancies. In total, 128 still frames were coded during the training phase (Set 1 in Table 1). Most of the discrepancies occurred when one or both of the coders experienced difficulty with distinguishing humans from stationary objects, such as trash-bins or mannequins. To solve this, the coders were allowed to look at different still frames obtained from the same video, to determine if an object was stationary (i.e., whether it moved between still frames). To assess whether two people were 1.5 meter apart, the coders were provided with examples of reference objects often visible on the still frames and their respective sizes (e.g., bikes, tiles on the street, sidewalk signs, benches, markings of a pedestrian crossing). The coding instructions were finalized after the training and applied in the next phase. The finalized coding instructions are provided in Supplemental Material 1.

\subsubsection{Inter-coder reliability}

To determine whether the human coding was sufficiently reliable to serve as a ground truth for the algorithm evaluation, a subset of the still frames (Set 2 in Table 1, $N=221$ frames) was double-coded by having the two human coders assess them independently. ${ }^{4}$

Inter-coder reliability was calculated for both the number of people and the number of social distancing violations $(<1.5$-meter proximity) that coders observed in the still frames. Both variables were treated as interval-level variables, for which inter-rater reliability can be determined with Krippendorff's alpha (Krippendorff, 2004; 2011). Krippendorff's alpha has a theoretical range between -1 (perfect disagreement between coders) and 1 (perfect agreement between coders), with 0 indicating the absence of any relation between the observations of the coders. The decision on a minimally acceptable level of alpha depends on the costs of drawing invalid conclusions. Krippendorff (2004:241) recommends to only

\footnotetext{
${ }^{4}$ It should be noted that a reliable measure of ground truth does not necessarily mean that that the measure is valid. If multiple coders share the same systematic bias, their estimates are reliable but not valid. Reliability is a weaker requirement than validity, as validity requires reliability.
} 
rely on variables with alpha reliabilities above .80, and to consider only variables with alpha between .67 and .80 for drawing tentative conclusions.

Table 2 reports the Krippendorff's alpha estimates and their 95\% confidence intervals for the coding. The alpha's reflect almost perfect agreement between the two coders for both the person counts (.98) and the violation counts (.93) and are well above the threshold of .80 recommended by Krippendorff (2004:241). This even holds for the lower boundaries of their 95\% confidence intervals (.97 and .90, respectively).

\section{INSERT TABLE 2}

Based on these results, we concluded that the human coding was sufficiently reliable to serve as ground truth for the evaluation of the algorithm. Consequently, the still frames in Set 3 were only coded by one of the coders (see Table 1).

\subsection{Development of the algorithm}

The task for the algorithm was the same as the task for the human coders and thus twofold: to detect all persons in the still frame and to identify all instances where two people were closer to each other than 1.5 meter. The first task, identifying persons, is the main element of existing algorithms for 'pedestrian detection.' These algorithms aim to identify pedestrians in traffic situations. Their current development is targeted towards the realization of self-driving vehicles, which must be able to identify objects in traffic and react adequately to them. Therefore, rather than reinvent the wheel, we decided to use an existing algorithm for pedestrian detection and extend it with a capacity to estimate distances between detected pedestrians.

\subsubsection{Programming the detector}

In the field of pedestrian detection, there are many different algorithms, and also many different annotated datasets that provide a ground truth for performance testing. The algorithm that was used in the present study builds on the Pedestron algorithm (Hasan et al., 2020), which is a deep learning algorithm that has been developed specifically for pedestrian detection and that performs well on multiple popular annotated datasets for pedestrian detection. We compared several instances of the Pedestron algorithm trained on different datasets, and decided to apply the version that was used in the Pedestrian Detection track of the WIDER Face and Person Challenge 2019 (an open competition between algorithms), as it provided the best results on the public datasets used in this challenge and on our test dataset.

Because the Pedestron algorithm only detects people, but not the distances between them, it had to be extended. Estimating a three-dimensional distance from a two-dimensional projection is not trivial. To assess the distance between two people, we estimated a linear regression model. This model was based on 
two simplifying assumptions. First, based on population statistics, we assumed that all persons are 1.70 meter tall. Second, we assumed that there is a linear relationship between the y-coordinate of the position of a person in a still frame and the size of that person depicted in the image. In other words, people closer to the camera appear larger in the image than people further away from the camera.

With information about the size of the detected persons from a large number of still frames (taking into account the different cameras, camera view angles, camera view directions, and camera zoom levels), we estimated a linear model that gave the relationship between the y-coordinate in an image and the height of a person. The model was calculated with the still frames (Set 2) plus, for each of these still frames, additional frames from the same video with 1.2 seconds interval. This linear model provided an estimate of the distance between two detected persons who are located perpendicular to the camera view (panel A in Figure 3). It is more difficult to determine the distance between persons in the direction of the camera view (panel B in Figure 3) because the underlying mathematical problem is underdetermined; there are more unknowns than there are data. ${ }^{5}$

Once estimates are determined for translating horizontal and vertical pixel counts to real-world distances, Pythagoras' theorem can be used to estimates the distance between two people in the most common situation, in which they are located neither completely perpendicular to the camera (beside each other), nor completely in the direction of the camera view (in front of and behind each other).

To optimize the estimation of this distance, we experimented with linear scaling based on the detected height of a person and the knowledge that the cameras were usually a couple of meters above the ground and looking down on people at an angle. The two scaling parameters that relate distance perpendicular to the camera view on the one hand and the distance in the direction of the camera view on the other hand, were refined by the programmer after manual inspection of still frames that were coded by the algorithm. The estimation of these two parameters is a one-off action that applies to the complete dataset, and not to every individual camera. It underlines that our algorithm cannot be used out-of-the-box for any situation involving distance measures between people: using it in other domains (e.g., with indoor footage from schools, hospitals, or other public places, or with footage from body-worn cameras) would likely require adjustments of these parameters.

\footnotetext{
${ }^{5}$ The unknowns are the position of the camera in real world coordinates, the orientation of the camera, and its focal length. For example, for a given person the same size in pixels in the image can be obtained by changing the distance to the camera or by changing the zoom level (focal length). From the picture there is no way to tell what is the contribution of the two to the actual size. Normally, these parameters can be detemined via manual calibration procedure but that is not possible in our use case because we had no control over the cameras. In addition, the parameters can change over time because most surveillance cameras are pan-tilt-zoom cameras.
} 


\section{INSERT FIGURE 3}

\subsubsection{Assess algorithm performance}

Because the ultimate visual task in our application is not to locate all people in a still frame but merely to count them (and similarly to count the number of distancing violations in the frame), we assessed the performance of the algorithm by relating the number of people and the number of social distancing violations detected by the algorithm with the number of people and violations detected by the human coders. In other words, we evaluate total numbers detected and ignore any potential differences between algorithm and human coders with respect to where precisely in the still frame peope were detected.

To assess performance, we used the Krippendorff's alpha, the same metric that we used to determine inter-coder reliability between the human coders. An advantage of using this metric not only for the intercoder reliabiliy but also for the algorithm performance, is that the metric can also be interpreted as a measure of human-algorithm reliability, and that we can use the same criterion for sufficient performance as we would use for human coders, i.e., the alpha threshold of .80 as recommended by Krippendorff (2004:241). The first version of the algorithm was evaluated using Set 2, with the assessments of the first coder used as ground truth. The second version of the algorithm was evaluated using both Set 2 (the results of which were input to the improvement) and Set 3 (which had not been used before, and was also coded by the first coder). The additional test on Set 3 was to make sure that the adaption made to the first version did not lead to overfitting, a situation in which an algorithm is tailored to perform high on a specific dataset but fails to generalize this high performance to other datasets.

\section{INSERT TABLE 3}

Table 3 summarizes the quantitative results in terms of Krippendorff's alpha. According to the top row in the table, which refers to numbers of people observed, the first version of the algorithm already performed above expectations. The estimated value of alpha is .85 , and even the lower boundary of the confidence interval is as high as .79. Although generalizability to other domains and datasets is never guaranteed, this result is hardly surprising because the algorithm was built on the already successful Pedestron algorithm. Because of the acceptable performance, we decided that the person detection element of the algorithm was not in need of further improvement. As a consequence, the second version of the algorithm was characterized by exactly the same alpha and confidence interval on Set 2 of the data, and by virtually the same alpha (and approximately the same confidence interval) on Set 3 of the data. The increased width of the confidence interval is the result of Set 3 being smaller $(N=201$ still frames) than Set $2(N=221$ still frames). 
We next address the results in the lower panel of Table 3, which summarizes the outcomes for the number of social distancing violations observed (i.e., $<1.5$-meter proximity count). The performance of the first version of the algorithm for detecting violations was much weaker than it was for detecting persons, as the estimated alpha was only .52, with the lower boundary of the confidence interval at .24 .

To improve the algorithm, the estimation of distance in the direction of the camera view was refined as discussed above and, additionally, the threshold for detecting distance violations was adjusted to be less conservative in the detection of social distancing violations. The second version of the algorithm was applied on the same benchmark data (Set 2).

The fifth row in Table 3 shows that, as a result of the adaptation, the Krippendorff's alpha raises to .85, which is excellent and approximately equal to the value for the person counts. ${ }^{6}$ The improved version of the algorithm, the second version, was subsequently applied to a fresh set of observations (Set 3, $N=205$ still frames). With this dataset, we confirmed a high agreement between the algorithm and the human coder in detecting social distancing violations, as expressed by the Krippendorff's alpha of .84, and presented in Table 3.

The results presented here are based on footage that was recorded in daylight. With a different sample of the footage (in the context of an evaluation of a curfew measure implemented, for details see Liebst, Appelman, Bernasco et al. 2021a), we also assessed the performance of the algorithm for detecting persons from footage recorded in darkness. The 'darkness' sample included all 420 still frames taken from 55 cameras in Amsterdam on Thursdays and Saturdays at 8 PM and at 9 PM in the period from November $12^{\text {th }}$ to November $28^{\text {th }}, 2020$. Sunset was between $4: 51 \mathrm{PM}$ (on November $12^{\text {th }}$ ) and 4:32PM (on November $28^{\text {th }}$ ). The results were indicative of high performance of the algorithm during darkness: The Krippendorff's alpha was .90 (95\% confidence interval .88-.93) for the comparison of the ratings by one human coder and version 2 of the algorithm. (The Krippendorff's alpha was .96 [95\% confidence interval .95-.97] for the comparison of the ratings by the two human coders.)

\subsection{Application of the algorithm}

After establishing that the algorithm performed satisfactorily, we applied it to measure social distancing compliance over the course of the pandemic. Here, we present estimates of both measures for just over a full year, from Thursday March 5, 2020 to Saturday March 27, 2021.

\footnotetext{
${ }^{6}$ For verification, we also determined the reliability measures between the second coder and both version of the algorithm on Set 2 of the data, which were similar to those of the first coder. For the person counts the alpha was .84 in both versions of the algorithm (.85 in case of the first coder). For the violation counts they were .57 with version 1 of the algorithm (.52 in case of coder 1$)$ and .83 with the second version of the algorithm (.84 in case of coder 1$)$.
} 


\subsubsection{Data collection}

From the Thursday and Saturday video recordings of 57 CCTV cameras in Amsterdam, still frames were extracted at every full hour from 9 AM to 8 PM. This scheme produced a total of 66,175 still frames that are automatically coded with the computer vision algorithm, counting both the numbers of persons present on the still frames and the detected number of distance violations.

In a perfect world, the observation scheme would produce 55 weeks $\times 2$ days $\times 12$ hours $\times 57$ cameras $=$ 95,760 still frames. For various technical and organizational limitations, a nontrivial proportion of these observations ( 31 percent) could not be processed and analyzed. First, for organizational reasons ${ }^{7}$ no recordings had been stored during 5 weeks in the Summer (July 23 to August 29, 2020), which amounts to 5 weeks $\times 2$ days $\times 12$ hours $\times 57$ cameras $=6,840$ still frames. Our estimates of social distancing compliance, therefore, contain a gap during this period. Second, during the first months of recording (March-May 2020) the video conversion procedure (from the proprietary G64X format to the open-source MP4 format) involved unrecoverable errors which resulted in the MP4 video files being truncated at some random point in time between $9 \mathrm{AM}$ to $8 \mathrm{PM}$. Third, throughout the full period, for technical reasons some observations were missing because the camera did not function on that particular day. While the first cause of missing observations has not been solved (so that the data contain a 5-week gap), the second and third causes were addressed by using a multiple imputation strategy that is described in the next section.

\subsubsection{Multiple imputation strategy}

The observations that are missing due to the three identified causes comprise 31 percent of the sample frame. The probability of a data frame to be missing is strongly and systematically related to the time of day (it increases over time from 9:00 to 8:00), and appears also related to the camera from which it was recorded and the date on which it was recorded, with a relatively large probability of missingness between March and June 2020 (and with certainty in the five-week summer gap). These relations are visualized in Figure S1 in Supplemental Material 4. Taking this into consideration, the missing values are imputed using multiple imputation (Rubin 1987). Multiple imputation replaces the dataset with a set of dataset copies, where in each copy the missing values have been replaced by alternative values that are sampled from a distribution, preserving uncertainty in the data. The multiple imputation procedure was implemented using the mi ce package (van Buuren 2018) for the R statistical software (R Development

\footnotetext{
${ }^{7}$ During this period, due to vacation leaves and sick leaves the police did not have the human resources available to copy, convert, and store the video recordings.
} 
Core Team 2012) after extended simulation tests using subsets of data. These tests and the setup of the multiple imputation procedure are described in Supplemental Material 4.

\subsubsection{Findings}

Figure 4 presents the observed development of the number of people on the street and the number of social distancing violations in Amsterdam over the course of the pandemic. The vertical axis represents the average observed number of people present per still frame, and the observed number of violations of the 1.5-meter directive per still frame. Each data point represents 1,368 still frames (57 cameras, 2 days per week and 12 hours per day). There are notable fluctuations throughout the study period, in both the number of people on the street and the number of contact moments.

Based on visual inspection, the number of people and the number of 1.5-meter contact moments appear to fluctuate in synchrony. This observation is confirmed by the correlation between persons and contact moments, which is .82, and still is .79 after correction for temporal autocorrelation and the inclusion of fixed effects for cameras. ${ }^{8}$ Although these are observational data that do not necessarily imply a causal relationship, they may signal that it is increasingly difficult to keep a 1.5-meter distance as crowds become larger in number, which could support crowd-control policies to reduce activity peaks and allow people to keep a distance, and might discourage strategies that make moral appeals to keep a distance from others. A more comprehensive analysis of this relation is described by (Liebst, Bernasco, EjbyeErnst et al. 2021b)

To contextualize the fluctuations in numbers of people on the street, we constructed a timeline indicating the strictness of the implemented COVID-19 social distancing measures during different periods. The strictness of these measures is represented by the background grey tones in Figure 4 . We distinguish between five levels: 'none or very few measures' (e.g., request to not shake hands), 'mild measures' (mandatory closing times for bars, limitations to the allowed number of visitors indoors), 'strict measures' (certain sectors of the economy closed down, limitations to sport events), 'lockdown' (schools closed, flow locations closed), and 'hard lockdown' (all non-essential shops closed).

\section{INSERT FIGURE 4}

\footnotetext{
${ }^{8}$ To correct for the temporally autocorrelated nature of the observations (two observations of the same camera close in time are more related than two observations distant in time), we first de-trended the observations by regressing them with OLS on the day of the week (either Thursday or Saturday), a second-order polynomial function of the date (day number and day number squared) and a second-order polynomial function of the hour of the day (hour and hour squared), and subsequently correlated the estimated residuals of the equations. This procedure removes a common time-trend from the correlations and yields a correlation coefficient of .81 . To remove in addition any between-camera variation from the estimate, we further added effects for each of the 57 cameras, yielding a correlation coefficient of .79.
} 
The first noticeable drop in the number of people on the street and the number of contact moments in Figure 4 is in the weeks prior to March 23, 2020, which coincides with the first announcements of social distancing directives by the Dutch government, which concerned general directives (e.g., avoid shaking hands, work from home). The 1.5-meter distance directive was only introduced March 15 and not sanctioned until March 23, suggesting that people started to avoid busy places already before the directives were officially implemented. This is in line with recent findings that show that during the pandemic, some behavioral changes preceded formal implementation (Allcott, Boxell, Conway et al. 2020, Chetty, Friedman, Hendren et al. 2020). They speak to the important role of voluntary action in reducing viral transmission rates (Brezinski, Deiana, Kecht et al. 2020), at least during the first stages of a pandemic.

After this initial drop, the activity level slowly increases over the following weeks until it reaches an average of 8.5 people per still frame at the end of the lockdown (the week of May 11, 2020). It remains quite high until a second drop in October, which is potentially related to a national holiday period.

A third noticeable drop appears in the week of December 14, 2020, a week after the so-called hard lockdown was enforced by the government. After this drop, the crowding and contact levels increased slowly over the following weeks. However, at the end of February there is a sudden increase, followed by a drop after two weeks. It seems plausible that temperature fluctuations play a role in these trends, as low temperatures may deter outdoor activity (average temperature in February 2021 was 4 degrees Celsius).

At the time of writing, the computer vision algorithm is still being used to monitor activity levels and distances between people, to regularly inform the municipality of Amsterdam about compliance with social distancing directives in public space (Bernasco, Liebst, van der Veen et al. 2021). They are also used in ongoing research addressing social distancing behavior during the COVID-19 pandemic, including a study on the effects of a night curfew (Liebst et al. 2021a).

\section{Looking forward}

In the preceding sections, we introduced computer vision to social scientists, we reviewed the extant literature on social signal processing and the recognition of persons, groups, and crowds, provided examples of studies that applied computer vision to address questions relevant to social scientists, and we gave a step-by-step example of how computer vision can be used to upscale empirical analysis of video data. In this concluding section, we attempt to look forward by discussing possible additional applications of our distancing model to sociologically relevant research questions, and by highlighting technical and ethical limitations to this methodology. 


\subsection{Contribution to sociological scholarship}

What exactly is the added value of measuring the behavioral displays of social distancing with a computer vision tool, rather than using a more traditional micro-sociological tool such as systematic on-site observations, as in the case of Collins (2020)? What is striking about Collins' study is that despite its ambition, it does not include direct records of social distancing behavior. Instead, Collins captures this behavior indirectly via counts of the greetings that people may perform when passing each other in a distanced manner, in addition to counts of mask-wearing. Probably, this reflects how social distancing behaviors is much more difficult, if not impossible, to record in a reliable manner when only having a single chance to observe this micro-detailed interaction (Philpot et al. 2019). This contrasts the greeting and mask-wearing behaviors, which are easier to record because they are less interactional, more static, and do not involve any metric dimension.

The playback function of recorded video data enables researchers to overcome such limitations of the human visual and cognitive systems and to process high volumes of data at high speed. The function of computer vision is to completely bypass the human coding process once we have ascertained that its performance is at least as good as human performance under optimal conditions (i.e., on recorded images by multiple coders, with rewinding and speed). This facilitates opportunities to better quantify phenomena and determine generalizable relationships between them. For example, it would enable large-scale data collection for cross-cultural comparisons, and link social distancing behaviors during the pandemic these to measures of cultural adherence to social norms (Gelfand, Jackson, Pan et al. 2021), collectivism (Hofstede 1980), authoritarianism (Gabennesch 1972), and preferred interpersonal distance (Gokmen, Turen, Erdem et al. 2020).

Aside from its application to COVID-19 related research, algorithms could be used to examine individual and cultural differences in distancing behavior. In his proxemic theory, Hall (1966:113-30) distinguished four categories of interpersonal distance that each reflected a different type of relationship between individuals: public distance among strangers, social distance maintained in formal interactions, personal distance among friends and relatives, and intimate distance among close friends, relatives, and romantic partners. He also theorized that the preferred degree of distance in interactions with others will depend on characteristics of the individual, dyad, and the broader social environment. Indeed, empirical work confirms that there are individual and dyadic differences with respect to age (Gérin-Lajoie, Richards and McFadyen 2006) and gender (Ozdemir 2008), as well as cross-national differences in people's preferences for personal space (Sorokowska et al. 2017). However, with some exceptions, most work in this area relies on small samples or projective measures of distancing. Video analysis in combination with computer vision methods would enable the large-scale observational assessment of this topic. 
Relatedly, computer vision could be used to examine in-group and out-group dynamics in distancing behavior. In an example of such work, Dietrich and Sands (2021) combined experimental and observational techniques to examine racial avoidance and found that pedestrians, particularly phenotypical non-black pedestrians, maintain relatively more distance from African American confederates than from non-Hispanic white confederates in public encounters on the sidewalks of New York City

Finally, algorithms such as the one developed in this study could be used to examine the contextdependency of distancing behavior. Structural features such as community disorder, racial composition, and crime rates have often been linked to feelings of safety and corresponding adjustments in selfprotective behaviors (Hale 1996, Liska, Lawrence and Sanchirico 1982). Computer vision has great potential for the large-scale examination of whether the distancing behavior of pedestrians varies across areas characterized by, for example, different types of land use, signs of physical and social disorder, and signs of territoriality.

\subsection{Challenges and limitations}

The exciting prospects of the field of computer vision should be considered alongside the various challenges that still remain. It should be acknowledged that video data analysis, and by implication the adoption of computer vision technology in it, holds the promise to advance only parts of sociological inquiry. The subjectively meaningful dimension of social interactions is arguably not the strong suite of a visually based analysis (Geertz 1973, though for a counterargument, see Katz 1999), and computer vision techniques should thus not be seen as alternatives but as additions to the existing methodological toolbox of sociologists.

Despite the quick development of camera technology, video recordings are not perfect. The accuracy of the detected events varies with camera angles, quality of the footage (e.g., number of pixels), illumination, and, for outdoor recordings, weather conditions (e.g., rain, snow). Efforts in the realm of social signal processing are even more sensitive to noise, occlusion, and other restrictions to the video quality than efforts toward group and crowd analysis. For example, to enable facial expression analysis, subjects need to be faced directly toward the camera, which is why this field is still heavily dependent on recordings obtained under lab-like conditions (Martinez 2019). Therefore, many advancements within social signal processing still have restricted real-world applicability.

Further, the communication of emotions may vary across cultures, individuals, and situations, which limits the generalizability of studies that infer affective states from facial movements and body postures (Barrett, Adolphs, Marsella et al. 2019, Kleinsmith and Bianchi-Berthouze 2013). 
For the recognition of groups and crowds, the detection and tracking of people remain challenging, especially in crowded scenes and in imagery with cluttered backgrounds: people might be indistinguishable from objects, or they might block the view on other individuals. Also, the dynamic nature of people's movements makes it difficult to estimate their future positions; people often change their speed to avoid collision with obstacles or other people in or out of view.

The detection of crowd abnormalities is challenging due to the context-dependent nature of such abnormalities (Wang and Loy 2017). For example, a person walking in the opposite direction of the crowd is a normal event in a shopping street, but can be considered an anomaly in a protest march. Because of this problem, many models on crowd analysis are scene-specific and would need to be retrained with new data to fit a different scene. Efforts are underway to enable scene-independent crowd analyses (Shao, Change Loy and Wang 2014, Yang, Cao, Wang et al. 2018), which would widen the application possibilities.

More generally, there is a lack of adequate training samples, synthetic as well as in-the-wild, which limits the generalization of the developed algorithms. But even if training samples would be abundant, our advice to social scientists using computer vision would be to always create at least a small training set, have it annotated by human coders and verify that the results of an automated ready-to-run algorithm align well with the human coding.

There are also ethical considerations that constrain the usefulness of video data (Legewie and Nassauer, 2018). First, most if not all video data will depict humans, and many of them have not explicitly agreed to be caught on camera. Video data are very difficult to make anonymous because the bodies of individuals are linked to their identities. Making them unrecognizable (e.g., by blurring their heads or upper bodies) may interfere with the aims of the research. Although recent evidence suggests that some anonymization methods hardly degrade the performance of computer vision (Klomp, van Rijn, Wijnhoven et al. 2021), considerations of privacy currently still often imply that the data must be processed in secure analytics labs, and that sharing of data (as advocated in calls for open science) is problematic (see Legewie and Nassauer, 2018 for a more extensive discussion). Whereas some studies have apparently circumvented this issue by using publicly available online recordings from video platforms like YouTube (e.g., Nassauer 2017, e.g., Nassauer 2018), this solution does not solve all ethical issues (Legewie and Nassauer, 2018).

Another ethical concern, unrelated to privacy, is the possibility that deep learning techniques are biased against groups in the population (e.g., adolescents, ethnic minorities) without the reasons of their biasedness being easily detectable, as they have become hard-wired in algorithms. Algorithm 
performance is ultimately judged against a ground truth defined by humans, and if human coders have biases, these biases become part of the annotated data on which algorithms are trained. Ultimately, these biases may also be acted upon when the algorithms are used for crowd control of other applications.

While the fear of being governed by algorithms that cannot motivate their judgments is usually phrased against algorithms that help to make decisions for governments and businesses, social scientists should also be aware of the possibility that computer vision algorithms contain biases and thereby might jeopardize the validity of their findings.

\subsection{The marriage of social science and computer vision}

An important conclusion of this study is that collaboration between social science and computer vision is not just feasible but can become symbiotic if social scientists clearly recognize the strengths and limitations of computer vision. In particular, social scientists should understand that computer vision is not an industry producing finished tools and solutions. It is a quickly developing scientific discipline that is driven by the same knowledge cycles and paradigm shifts other sciences go through. Social scientists should be prepared to take part in the development of these solutions. They should have patience but also contribute to posing the right questions and to solving challenges. What social scientists bring to the table is the ability to formulate challenging questions motivated by social theory. However, it should also be mentioned that, besides interdisciplinary collaboration, there are also other options available to social scientists for engaging with CV, option that do not necessarily involve technical know-how. We would like to emphasize, however, that social scientists do not need a degree in computer vision to use it in their research. In fact, it may be more efficient to collaborate with computer vision researchers than try to become one of them.

\section{References}

Allcott, H. , L. Boxell, J. Conway, B. Ferguson, M. Gentzkow and B. Goldman. 2020. "What Explains Temporal and Geographic Variation in the Early Us Coronavirus Pandemic?" SSRN 3610422 Retrieved: posted 2020 May 25, revised 2021 Jan 2 (http://dx.doi.org/10.2139/ssrn.3610422).

Almagbile, Ali. 2019. "Estimation of Crowd Density from Uavs Images Based on Corner Detection Procedures and Clustering Analysis." Geo-spatial Information Science 22(1):23-34. doi: 10.1080/10095020.2018.1539553.

Baig, Mirza Waqar, Emilia I. Barakova, Lucio Marcenaro, Matthias Rauterberg and Carlo S. Regazzoni. 2014. "Crowd Emotion Detection Using Dynamic Probabilistic Models." Pp. 328-37, From Animals to Animats 13. Cham: Springer International Publishing.

Barrett, Lisa Feldman, Ralph Adolphs, Stacy Marsella, Aleix M. Martinez and Seth D. Pollak. 2019. "Emotional Expressions Reconsidered: Challenges to Inferring Emotion from Human Facial Movements." Psychological Science in the Public Interest 20(1):1-68. doi: $10.1177 / 1529100619832930$. 
Bartlett, Marian Stewart, Gwen C. Littlewort, Mark G. Frank and Kang Lee. 2014. "Automatic Decoding of Facial Movements Reveals Deceptive Pain Expressions." Current Biology 24(7):738-43. doi: 10.1016/j.cub.2014.02.009.

Bauch, Chris T. and Alison P. Galvani. 2013. "Social Factors in Epidemiology." Science 342(6154):4749. doi: $10.1126 /$ science. 1244492 .

Bernasco, Wim, Lasse Suonperä Liebst, Thomas van der Veen, Nigel van Herwijnen, Joska Appelman, Josephine Thomas, Kiki Bijleveld, Dennis Koelma, Peter Ejbye-Ernst, Evelien Hoeben, Cees Snoek and Marie Rosenkrantz Lindegaard. 2021. "One Year of Social Distancing Behavior on the Streets of Amsterdam." Retrieved: March 10, 2021.

Black, Matthew P., Athanasios Katsamanis, Brian R. Baucom, Chi-Chun Lee, Adam C. Lammert, Andrew Christensen, Panayiotis G. Georgiou and Shrikanth S. Narayanan. 2013. "Toward Automating a Human Behavioral Coding System for Married Couples' Interactions Using Speech Acoustic Features." Speech Communication 55(1):1-21. doi: 10.1016/j.specom.2011.12.003.

Bogardus, Emory S. 1933. "A Social Distance Scale." Sociology \& Social Research 17:265-71.

Brezinski, Adam, Guido Deiana, Valentin Kecht and David Van Dijcke. 2020. "The Covid-19 Pandemic: Government Vs. Community Action across the United States." (https://www.inet.ox.ac.uk/files/BrzezinskiKechtDeianaVanDijcke_18042020_CEPR_2.pdf).

Bruer, Kaila C., Sarah Zanette, Xiao Pan Ding, Thomas D. Lyon and Kang Lee. 2020. "Identifying Liars through Automatic Decoding of Children's Facial Expressions." Child Development 91(4):e995e1011. doi: 10.1111/cdev.13336.

Burgoon, J.K., N. Magnenat-Thalmann, M. Pantic and A. Vinciarelli, eds. 2017. Social Signal Processing. Cambridge: Cambridge University Press. (https://www.cambridge.org/core/books/social-signalprocessing/834565FD44AED8C4FB112A6A8DE54318).

Canedo, Daniel and António J. R. Neves. 2019. "Facial Expression Recognition Using Computer Vision: A Systematic Review." Applied Sciences 9(21):4678. doi: 10.3390/app9214678.

Cao, Jiale, Yanwei Pang, Jin Xie, Fahad Shahbaz Khan and Ling Shao. 2021. "From Handcrafted to Deep Features for Pedestrian Detection: A Survey." IEEE Transactions on Pattern Analysis and Machine Intelligence:1-1. doi: 10.1109/TPAMI.2021.3076733.

Cao, Zhe, Gines Hidalgo, Tomas Simon, Shih-En Wei and Yaser Sheikh. 2019. "Openpose: Realtime Multi-Person 2d Pose Estimation Using Part Affinity Fields." IEEE Transactions on Pattern Analysis and Machine Intelligence 43(1):172-86.

Chang, Ming-Ching, Nils Krahnstoever, Sernam Lim and Ting Yu. 2010. "Group Level Activity Recognition in Crowded Environments across Multiple Cameras." Pp. 56-63 in 2010 7th IEEE International Conference on Advanced Video and Signal Based Surveillance.

Chen, Jixu, Ming-Ching Chang, Tai-Peng Tian, Ting Yu and Peter Tu. 2015. "Bridging Computer Vision and Social Science: A Multi-Camera Vision System for Social Interaction Training Analysis." Pp. 823-26 in 2015 IEEE International Conference on Image Processing (ICIP).

Chetty, Raj, John N. Friedman, Nathaniel Hendren and Michael Stepner. 2020. "[Preprint] How Did Covid-19 and Stabilization Policies Affect Spending and Employment? A New Real-Time Economic Tracker Based on Private Sector Data." (http://www.nber.org/papers/w27431.pdf).

Collins, Randall. 2020. "Social Distancing as a Critical Test of the Micro-Sociology of Solidarity." American Journal of Cultural Sociology 8(3):477-97. doi: 10.1057/s41290-020-00120-z.

Conigliaro, Davide, Paolo Rota, Francesco Setti, Chiara Bassetti, Nicola Conci, Nicu Sebe and Marco Cristani. 2015. "The S-Hock Dataset: Analyzing Crowds at the Stadium." Pp. 2039-47 in Proceedings of the IEEE Conference on Computer Vision and Pattern Recognition.

Cruz, Marcos and Javier González-Villa. 2021. "Unbiased Population Size Estimation on Still Gigapixel Images." Sociological Methods \& Research 50(2):627-48. doi: 10.1177/0049124118799373.

Dietrich, Bryce J. and Melissa Sands. 2021. "Seeing Racial Avoidance on City Streets." http://www.brycejdietrich.com/files/working_papers/DietrichSands.pdf. 
Dutta, Abhishek and Andrew Zisserman. 2019. "The Via Annotation Software for Images, Audio and Video." Paper presented at the Proceedings of the 27th ACM International Conference on Multimedia, Nice, France (https://doi-org.vu-nl.idm.oclc.org/10.1145/3343031.3350535).

Fong, Min W, Huizhi Gao, Jessica Y Wong, Jingyi Xiao, Eunice YC Shiu, Sukhyun Ryu and Benjamin J Cowling. 2020. "Nonpharmaceutical Measures for Pandemic Influenza in Nonhealthcare Settings - Social Distancing Measures." Emerging infectious diseases 26(5):976-84. doi: 10.3201/eid2605.190995.

Forsyth, David A and Jean Ponce. 2012. Computer Vision: A Modern Approach: Pearson.

Gabennesch, Howard. 1972. "Authoritarianism as World View." American Journal of Sociology 77(5):857-75. doi: 10.1086/225228.

Gallupe, Owen, John McLevey and Sarah Brown. 2019. "Selection and Influence: A Meta-Analysis of the Association between Peer and Personal Offending." Journal of Quantitative Criminology 35(2):313-35. doi: 10.1007/s10940-018-9384-y.

Ge, Weina, Robert T. Collins and R. Barry Ruback. 2012. "Vision-Based Analysis of Small Groups in Pedestrian Crowds." IEEE Transactions on Pattern Analysis and Machine Intelligence 34(5):1003-16. doi: 10.1109/TPAMI.2011.176.

Gebru, Timnit, Jonathan Krause, Yilun Wang, Duyun Chen, Jia Deng, Erez Lieberman Aiden and Li FeiFei. 2017. "Using Deep Learning and Google Street View to Estimate the Demographic Makeup of Neighborhoods across the United States." Proceedings of the National Academy of Sciences 114(50):13108-13. doi: 10.1073/pnas.1700035114.

Geertz, Clifford. 1973. The Interpretation of Cultures: Selected Essays. . New York, NY: Basic Books.

Gelfand, Michele J., Joshua Conrad Jackson, Xinyue Pan, Dana Nau, Dylan Pieper, Emmy Denison, Munqith Dagher, Paul A. M. Van Lange, Chi-Yue Chiu and Mo Wang. 2021. "The Relationship between Cultural Tightness-Looseness and Covid-19 Cases and Deaths: A Global Analysis." The Lancet Planetary Health 5(3):e135-e44. doi: 10.1016/S2542-5196(20)30301-6.

Gérin-Lajoie, Martin, Carol L. Richards and Bradford J. McFadyen. 2006. "The Circumvention of Obstacles During Walking in Different Environmental Contexts: A Comparison between Older and Younger Adults." Gait \& Posture 24(3):364-69. doi: 10.1016/j.gaitpost.2005.11.001.

Goffman, Irving. 1971. Relations in Public: Microstudies of the Public Order. New York, NY: Basic Books.

Gokmen, Yunus, Ufuk Turen, Haluk Erdem and İsmail Tokmak. 2020. "National Preferred Interpersonal Distance Curbs the Spread of Covid-19: A Cross-Country Analysis." Disaster Medicine and Public Health Preparedness:1-7. doi: 10.1017/dmp.2020.295.

Goodfellow, Ian, Yoshua Bengio and Aaron Courville. 2016. Deep Learning: MIT Press.

Grant, Jason M. and Patrick J. Flynn. 2017. "Crowd Scene Understanding from Video: A Survey." ACM Trans. Multimedia Comput. Commun. Appl. 13(2):Article 19. doi: 10.1145/3052930.

Hale, Chris. 1996. "Fear of Crime: A Review of the Literature." International Review of Victimology 4(2):79-150.

Hall, Edward T. 1966. The Hidden Dimension. Garden City, NY: Doubleday.

Haque, Mahfuzul and Manzur Murshed. 2010. "Panic-Driven Event Detection from Surveillance Video Stream without Track and Motion Features." Pp. 173-78 in 2010 IEEE International Conference on Multimedia and Expo.

Harikrishnan, J., A. Sudarsan, A. Sadashiv and R. A. S. Ajai. 2019. "Vision-Face Recognition Attendance Monitoring System for Surveillance Using Deep Learning Technology and Computer Vision."

Pp. 1-5 in 2019 International Conference on Vision Towards Emerging Trends in Communication and Networking (ViTECoN).

Hasan, Irtiza, Shengcai Liao, Jinpeng Li, Saad Ullah Akram and Ling Shao. 2020. "Generalizable Pedestrian Detection: The Elephant in the Room." in IEEE/CVF Conference on Computer Vision and Pattern Recognition.

Herath, Samitha, Mehrtash Harandi and Fatih Porikli. 2017. "Going Deeper into Action Recognition: A Survey." Image and Vision Computing 60:4-21. doi: 10.1016/j.imavis.2017.01.010. 
Hofstede, Geert. 1980. Culture's Consequences: International Differences in Work-Related Values. Beverley Hills, CA: Sage.

Hu, Yanghui, Jun Zhang, Weiguo Song and Nikolai W. F. Bode. 2021. "Social Groups Barely Change the Speed-Density Relationship in Unidirectional Pedestrian Flow, but Affect Operational Behaviours." Safety Science 139:105259. doi: 10.1016/j.ssci.2021.105259.

Huang, Yingjing, Teng Fei, Mei-Po Kwan, Yuhao Kang, Jun Li, Yizhuo Li, Xiang Li and Meng Bian. 2020. "Gis-Based Emotional Computing: A Review of Quantitative Approaches to Measure the Emotion Layer of Human-Environment Relationships." ISPRS International Journal of GeoInformation 9(9):551.

Hung, Hayley, Dinesh Jayagopi, Chuohao Yeo, Gerald Friedland, Sileye Ba, Jean-Marc Odobez, Kannan Ramchandran, Nikki Mirghafori and Daniel Gatica-Perez. 2007. "Using Audio and Video Features to Classify the Most Dominant Person in a Group Meeting." Paper presented at the Proceedings of the 15th ACM international conference on Multimedia, Augsburg, Germany (https://doi-org.vu-nl.idm.oclc.org/10.1145/1291233.1291423).

Hung, Hayley. and Daniel Gatica-Perez. 2010. "Estimating Cohesion in Small Groups Using AudioVisual Nonverbal Behavior." IEEE Transactions on Multimedia 12(6):563-75. doi: 10.1109/TMM.2010.2055233.

Ivaniushina, Valeria and Vera Titkova. 2021. "Peer Influence in Adolescent Drinking Behavior: A MetaAnalysis of Stochastic Actor-Based Modeling Studies." PLoS ONE 16(4):e0250169. doi: 10.1371/journal.pone.0250169.

Jayagopi, D.B., H. Hung, C. Yeo and D. Gatica-Perez. 2009. "Modeling Dominance in Group Conversations Using Nonverbal Activity Cues." IEEE Transactions on Audio, Speech, and Language Processing 17(3):501-13. doi: 10.1109/TASL.2008.2008238.

Jerolmack, Colin and Shamus Khan. 2014. "Talk Is Cheap:Ethnography and the Attitudinal Fallacy." Sociological Methods \& Research 43(2):178-209. doi: 10.1177/0049124114523396.

Kantharaju, Reshmashree B, Caroline Langlet, Mukesh Barange, Chloé Clavel and Catherine Pelachaud, I. 2020. "Multimodal Analysis of Cohesion in Multi-Party Interactions." Paper presented at the LREC, 2020, Marseille, France (https://hal.archives-ouvertes.fr/hal-02953469).

Katz, Jack. 1999. How Emotions Work: University of Chicago Press.

Kendon, Adam. 1990. Conducting Interaction: Patterns of Behavior in Focused Encounters. New York, NY: Cambridge University Press.

Kleinsmith, Andrea and Nadia Bianchi-Berthouze. 2013. "Affective Body Expression Perception and Recognition: A Survey." IEEE Transactions on Affective Computing 4(1):15-33. doi: 10.1109/TAFFC.2012.16.

Klomp, Sander R., Matthew van Rijn, Rob G.J. Wijnhoven, Cees G.M. Snoek and Peter H.N. de With. 2021. "Safe Fakes: Evaluating Face Anonymizers for Face Detectors." arXiv preprint arXiv:2004.11430.

Krippendorff, Klaus. 2004. Content Analysis: An Introduction to Its Methodology. Thousand Oaks, CA: Sage.

LeCun, Yann, Yoshua Bengio and Geoffrey Hinton. 2015. "Deep Learning." Nature 521(7553):436-44. doi: $10.1038 /$ nature 14539 .

Li, Junnan, Yongkang Wong, Qi Zhao and Mohan S. Kankanhalli. 2020. "Visual Social Relationship Recognition." International Journal of Computer Vision 128(6):1750-64. doi: 10.1007/s11263020-01295-1.

Li, Rui, Jared Curhan and Mohammed Hoque. 2018. "Understanding Social Interpersonal Interaction Via Synchronization Templates of Facial Events." Proceedings of the AAAI Conference on Artificial Intelligence 32(1).

Liebst, Lasse Suonperä, Joska Appelman, Wim Bernasco, Evelien Hoeben, Cees Snoek, Dennis Koelma and Marie Rosenkrantz Lindegaard. 2021a. "Behavioral Compliance with Curfew Measure During Lockdown: Results of the Nscr Amsterdam Curfew Study (Pre-Print, Version 3)." (https://osf.io/7ek9d/). 
Liebst, Lasse Suonperä, Wim Bernasco, Peter Ejbye-Ernst, Thomas van der Veen, Nigel van Herwijnen, Dennis Koelma, Cees Snoek and Marie Rosenkrantz Lindegaard. 2021b. "Social Distancing Violations as a Situational Correlate of Crowding: Evidence from a Large-Scale Video Observational Study of Public Behavior During the Covid-19 Pandemic (Pre-Print)." (https://osf.io/7ek9d/).

Lindegaard, Marie Rosenkrantz and Wim Bernasco. 2018. "Lessons Learned from Crime Caught on Camera." Journal of Research in Crime and Delinquency 55(1):155-86. doi: $10.1177 / 0022427817727830$.

Liska, Allen E., Joseph J. Lawrence and Andrew Sanchirico. 1982. "Fear of Crime as a Social Fact*." Social Forces 60(3):760-70. doi: 10.1093/sf/60.3.760.

Liu, Yu, Yihong Yuan and Fan Zhang. 2020. "Mining Urban Perceptions from Social Media Data." Journal of Spatial Information Science 2020(20):51-55.

Lv, Jinna, Wu Liu, Lili Zhou, Bin Wu and Huadong Ma. 2018. "Multi-Stream Fusion Model for Social Relation Recognition from Videos." Pp. 355-68, MultiMedia Modeling. Cham: Springer International Publishing.

Martinez, Aleix M. 2019. "The Promises and Perils of Automated Facial Action Coding in Studying Children's Emotions." Developmental Psychology 55(9):1965-81. doi: 10.1037/dev0000728.

Mohammadi, Sadegh, Hamed K. Galoogahi, Alessandro Perina and Vittorio Murino. 2017. "Chapter 11 Physics-Inspired Models for Detecting Abnormal Behaviors in Crowded Scenes." Pp. 253-72 in Group and Crowd Behavior for Computer Vision, edited by V. Murino, M. Cristani, S. Shah and S. Savarese: Academic Press.

Morrison, Christopher, Juliet P. Lee, Paul J. Gruenewald and Christina Mair. 2016. "The Reliability of Naturalistic Observations of Social, Physical and Economic Environments of Bars." Addiction Research \& Theory 24(4):330-40. doi: 10.3109/16066359.2016.1145674.

Moussaïd, Mehdi, Niriaska Perozo, Simon Garnier, Dirk Helbing and Guy Theraulaz. 2010. "The Walking Behaviour of Pedestrian Social Groups and Its Impact on Crowd Dynamics." PLoS ONE 5(4):e10047. doi: 10.1371/journal.pone.0010047.

Murino, Vittorio, Marco Cristani, Shishir Shah and Silvio Savarese. 2017a. "The Group and Crowd Analysis Interdisciplinary Challenge." Pp. 1-11 in Group and Crowd Behavior for Computer Vision, edited by V. Murino, M. Cristani, S. Shah and S. Savarese. London: Academic Press.

Murino, Vittorio, Marco Cristani, Shishir Shah and Silvio Savarese. 2017b. Group and Crowd Behavior for Computer Vision. London: Academic Press.

Nassauer, Anne. 2017. "How Robberies Succeed or Fail: Analyzing Crime Caught on CCTV." Journal of Research in Crime and Delinquency 54.

Nassauer, Anne. 2018. "How Robberies Succeed or Fail: Analyzing Crime Caught on CCTV." Journal of Research in Crime and Delinquency 55(1):125-54. doi: 10.1177/0022427817715754.

Nassauer, Anne and Nicolas M. Legewie. 2021. "Video Data Analysis: A Methodological Frame for a Novel Research Trend." Sociological Methods \& Research 50(1):135-74. doi: $10.1177 / 0049124118769093$.

Nayak, Rashmiranjan, Umesh Chandra Pati and Santos Kumar Das. 2021. "A Comprehensive Review on Deep Learning-Based Methods for Video Anomaly Detection." Image and Vision Computing 106:104078. doi: 10.1016/j.imavis.2020.104078.

Ozdemir, Aydin. 2008. "Shopping Malls: Measuring Interpersonal Distance under Changing Conditions and across Cultures." Field Methods 20(3):226-48. doi: 10.1177/1525822x08316605.

Philpot, Richard, Lasse Suonperä Liebst, Kim Kristian Møller, Marie Rosenkrantz Lindegaard and Mark Levine. 2019. "Capturing Violence in the Night-Time Economy: A Review of Established and Emerging Methodologies." Aggression and violent behavior 46:56-65. doi: 10.1016/j.avb.2019.02.004.

Pouw, Caspar A. S., Federico Toschi, Frank van Schadewijk and Alessandro Corbetta. 2020. "Monitoring Physical Distancing for Crowd Management: Real-Time Trajectory and Group Analysis." PLoS ONE 15(10):e240963. doi: 10.1371/journal.pone.0240963. 
R Development Core Team. 2012. R: A Language and Environment for Statistical Computing. Vienna, Austria: R Foundation for Statistical Computing

Rahim, Adina, Ayesha Maqbool and Tauseef Rana. 2021. "Monitoring Social Distancing under Various Low Light Conditions with Deep Learning and a Single Motionless Time of Flight Camera." PLoS ONE 16(2):e0247440. doi: 10.1371/journal.pone.0247440.

Rodriguez, Mikel, Josef Sivic and Ivan Laptev. 2017. "Chapter 5 - the Analysis of High Density Crowds in Videos." Pp. 89-113 in Group and Crowd Behavior for Computer Vision, edited by V. Murino, M. Cristani, S. Shah and S. Savarese: Academic Press.

Roman, David Gabriel Choqueluque and Guillermo Cámara Chávez. 2020. "Violence Detection and Localization in Surveillance Video." Pp. 248-55 in 2020 33rd SIBGRAPI Conference on Graphics, Patterns and Images (SIBGRAPI).

Roy, Debaditya and Krishna Mohan C. 2018. "Snatch Theft Detection in Unconstrained Surveillance Videos Using Action Attribute Modelling." Pattern Recognition Letters 108:56-61. doi: 10.1016/j.patrec.2018.03.004.

Rubin, Donald B. 1987. Multiple Imputation for Nonresponse in Surveys. New York: Wiley \& Sons.

Sanchez-Cortes, Dairazalia, Oya Aran, Marianne Schmid Mast and Daniel Gatica-Perez. 2012. "A Nonverbal Behavior Approach to Identify Emergent Leaders in Small Groups." IEEE Transactions on Multimedia 14(3):816-32. doi: 10.1109/TMM.2011.2181941.

Setti, Francesco, Chris Russell, Chiara Bassetti and Marco Cristani. 2015. "F-Formation Detection: Individuating Free-Standing Conversational Groups in Images." PLoS ONE 10(5):e0123783. doi: 10.1371/journal.pone.0123783.

Shao, Jing, Chen Change Loy and Xiaogang Wang. 2014. "Scene-Independent Group Profiling in Crowd." Pp. 2219-26 in Proceedings of the IEEE Conference on Computer Vision and Pattern Recognition.

Shia, Wei-Chung and Dar-Ren Chen. 2021. "Classification of Malignant Tumors in Breast Ultrasound Using a Pretrained Deep Residual Network Model and Support Vector Machine." Computerized Medical Imaging and Graphics 87:101829. doi: 10.1016/j.compmedimag.2020.101829.

Sigurdsson, Gunnar A, Olga Russakovsky and Abhinav Gupta. 2017. "What Actions Are Needed for Understanding Human Actions in Videos?" Pp. 2137-46 in Proceedings of the IEEE international conference on computer vision.

Small, Mario L. and Jenna M. Cook. 2021. "Using Interviews to Understand Why: Challenges and Strategies in the Study of Motivated Action." Sociological Methods \& Research:0049124121995552. doi: 10.1177/0049124121995552.

Sodemann, Angela A., Matthew P. Ross and Brett J. Borghetti. 2012. "A Review of Anomaly Detection in Automated Surveillance." IEEE Transactions on Systems, Man, and Cybernetics, Part C (Applications and Reviews) 42(6):1257-72. doi: 10.1109/TSMCC.2012.2215319.

Solera, Francesco, Simone Calderara and Rita Cucchiara. 2017. "From Groups to Leaders and Back: Exploring Mutual Predictability between Social Groups and Their Leaders." Pp. 161-82 in Group and Crowd Behavior for Computer Vision, edited by V. Murino, M. Cristani, S. Shah and S. Savarese: Academic Press.

Sorokowska, Agnieszka, Piotr Sorokowski, Peter Hilpert, Katarzyna Cantarero, Tomasz Frackowiak, Khodabakhsh Ahmadi, Ahmad M. Alghraibeh, Richmond Aryeetey, Anna Bertoni, Karim Bettache, Sheyla Blumen, Marta Błażejewska, Tiago Bortolini, Marina Butovskaya, Felipe Nalon Castro, Hakan Cetinkaya, Diana Cunha, Daniel David, Oana A. David, Fahd A. Dileym, Alejandra del Carmen Domínguez Espinosa, Silvia Donato, Daria Dronova, Seda Dural, Jitka Fialová, Maryanne Fisher, Evrim Gulbetekin, Aslıhan Hamamcıoğlu Akkaya, Ivana Hromatko, Raffaella Iafrate, Mariana Iesyp, Bawo James, Jelena Jaranovic, Feng Jiang, Charles Obadiah Kimamo, Grete Kjelvik, Firat Koç, Amos Laar, Fívia de Araújo Lopes, Guillermo Macbeth, Nicole M. Marcano, Rocio Martinez, Norbert Mesko, Natalya Molodovskaya, Khadijeh Moradi, Zahrasadat Motahari, Alexandra Mühlhauser, Jean Carlos Natividade, Joseph Ntayi, Elisabeth Oberzaucher, Oluyinka Ojedokun, Mohd Sofian Bin Omar-Fauzee, Ike E. Onyishi, Anna 
Paluszak, Alda Portugal, Eugenia Razumiejczyk, Anu Realo, Ana Paula Relvas, Maria Rivas, Muhammad Rizwan, Svjetlana Salkičević, Ivan Sarmány-Schuller, Susanne Schmehl, Oksana Senyk, Charlotte Sinding, Eftychia Stamkou, Stanislava Stoyanova, Denisa Šukolová, Nina Sutresna, Meri Tadinac, Andero Teras, Edna Lúcia Tinoco Ponciano, Ritu Tripathi, Nachiketa Tripathi, Mamta Tripathi, Olja Uhryn, Maria Emília Yamamoto, Gyesook Yoo and John D. Pierce. 2017. "Preferred Interpersonal Distances: A Global Comparison." Journal of CrossCultural Psychology 48(4):577-92. doi: 10.1177/0022022117698039.

Stephens-Fripp, Benjamin, Fazel Naghdy, David Stirling and Golshah Naghdy. 2017. "Automatic Affect Perception Based on Body Gait and Posture: A Survey." International Journal of Social Robotics 9(5):617-41. doi: 10.1007/s12369-017-0427-6.

Stipancic, Joshua, Sohail Zangenehpour, Luis Miranda-Moreno, Nicolas Saunier and Marie-Axelle Granié. 2016. "Investigating the Gender Differences on Bicycle-Vehicle Conflicts at Urban Intersections Using an Ordered Logit Methodology." Accident Analysis \& Prevention 97:19-27. doi: 10.1016/j.aap.2016.07.033.

Sultani, Waqas, Chen Chen and Mubarak Shah. 2018. "Real-World Anomaly Detection in Surveillance Videos." Pp. 6479-88 in Proceedings of the IEEE conference on computer vision and pattern recognition.

Sun, Qianru, Bernt Schiele and Mario Fritz. 2017. "A Domain Based Approach to Social Relation Recognition." Pp. 3481-90 in Proceedings of the IEEE Conference on Computer Vision and Pattern Recognition.

Szeliski, Richard. 2011. Computer Vision: Algorithms and Applications: Springer Science \& Business Media.

Torres, Michelle and Francisco Cantú. 2021. "Learning to See: Convolutional Neural Networks for the Analysis of Social Science Data." Political Analysis:1-19. doi: 10.1017/pan.2021.9.

Tran, Khai N, Apurva Bedagkar-Gala, Ioannis A Kakadiaris and Shishir K Shah. 2013. "Social Cues in Group Formation and Local Interactions for Collective Activity Analysis." Pp. 539-48 in VISAPP (1).

Tran, Minh, Taylan Sen, Kurtis Haut, Mohammad Rafayet Ali and Mohammad Ehsan Hoque. 2020. "Are You Really Looking at Me? A Feature-Extraction Framework for Estimating Interpersonal Eye Gaze from Conventional Video." IEEE Transactions on Affective Computing:1-1. doi: 10.1109/TAFFC.2020.2979440.

Tripathi, Rajesh Kumar, Anand Singh Jalal and Subhash Chand Agrawal. 2018. "Suspicious Human Activity Recognition: A Review." Artificial Intelligence Review 50(2):283-339. doi: 10.1007/s10462-017-9545-7.

Turner, Jonathan H. 2010. Theoretical Principles of Sociology. Volume 2: Microdynamics., Vol. 2. London: Sage.

Tzelepis, Christos, Zhigang Ma, Vasileios Mezaris, Bogdan Ionescu, Ioannis Kompatsiaris, Giulia Boato, Nicu Sebe and Shuicheng Yan. 2016. "Event-Based Media Processing and Analysis: A Survey of the Literature." Image and Vision Computing 53:3-19. doi: 10.1016/j.imavis.2016.05.005.

Valstar, Michel F, Hatice Gunes and Maja Pantic. 2007. "How to Distinguish Posed from Spontaneous Smiles Using Geometric Features." Pp. 38-45 in Proceedings of the 9th international conference on Multimodal interfaces.

van Buuren, Stef. 2018. Flexible Imputation of Missing Data. Second Edition. Boca Raton, FL: CRC Press.

Varghese, Elizabeth B. and Sabu M. Thampi. 2018. "A Deep Learning Approach to Predict Crowd Behavior Based on Emotion." Pp. 296-307, Smart Multimedia. Cham: Springer International Publishing.

Vascon, Sebastiano and Loris Bazzani. 2017. "Chapter 3 - Group Detection and Tracking Using Sociological Features." Pp. 29-66 in Group and Crowd Behavior for Computer Vision, edited by V. Murino, M. Cristani, S. Shah and S. Savarese: Academic Press. 
Veltmeijer, E. A., C. Gerritsen and K. Hindriks. 2021. "Automatic Emotion Recognition for Groups: A Review." IEEE Transactions on Affective Computing:1-1. doi: 10.1109/TAFFC.2021.3065726.

Vinciarelli, Alessandro, Maja Pantic and Hervé Bourlard. 2009. "Social Signal Processing: Survey of an Emerging Domain." Image and Vision Computing 27(12):1743-59. doi: 10.1016/j.imavis.2008.11.007.

Walocha, Fabian, Lucien Maman, Mohamed Chetouani and Giovanna Varni. 2020. "Modeling Dynamics of Task and Social Cohesion from the Group Perspective Using Nonverbal Motion CaptureBased Features." Paper presented at the Companion Publication of the 2020 International Conference on Multimodal Interaction, Virtual Event, Netherlands (https://doi-org.vunl.idm.oclc.org/10.1145/3395035.3425963).

Wang, Xiaogang and Chen-Change Loy. 2017. "Chapter 10 - Deep Learning for Scene-Independent Crowd Analysis." Pp. 209-52 in Group and Crowd Behavior for Computer Vision, edited by V. Murino, M. Cristani, S. Shah and S. Savarese: Academic Press.

Wikström, Per-Olof H., Dietrich Oberwittler, Kyle Treiber and Beth Hardie. 2012. Breaking Rules: The Social and Situational Dynamics of Young People's Urban Crime Oxford: Oxford University Press.

Won, Andrea Stevenson, Jeremy N. Bailenson and Joris H. Janssen. 2014. "Automatic Detection of Nonverbal Behavior Predicts Learning in Dyadic Interactions." IEEE Transactions on Affective Computing 5(2):112-25. doi: 10.1109/TAFFC.2014.2329304.

World Health Organization. 2009, "Pandemic Influenza Prevention and Mitigation in Low Resource Communities". Retrieved March 29, 2020, 2020 (https://www.who.int/csr/resources/publications/swineflu/low resource measures/en/).

Yang, Biao, Jin-Meng Cao, Nan Wang, Yu-Yu Zhang and Guo-Zeng Cui. 2018. "Cross-Scene Counting Based on Domain Adaptation-Extreme Learning Machine." IEEE Access 6:17029-38. doi: 10.1109/ACCESS.2018.2800688.

Yang, Yan, Md Zakir Hossain, Tom Gedeon and Shafin Rahman. 2020. "Realsmilenet: A Deep End-toEnd Network for Spontaneous and Posed Smile Recognition." in Proceedings of the Asian Conference on Computer Vision.

Yu, Ting, Ser-Nam Lim, Kedar Patwardhan and Nils Krahnstoever. 2009. "Monitoring, Recognizing and Discovering Social Networks." Pp. 1462-69 in 2009 IEEE Conference on Computer Vision and Pattern Recognition.

Zanette, Sarah, Xiaoqing Gao, Megan Brunet, Marian Stewart Bartlett and Kang Lee. 2016. "Automated Decoding of Facial Expressions Reveals Marked Differences in Children When Telling Antisocial Versus Prosocial Lies." Journal of Experimental Child Psychology 150:165-79. doi: 10.1016/j.jecp.2016.05.007.

Zhang, Kun, Dongzhi Chen and Chunlin Li. 2020. "How Are Tourists Different? - Reading Geo-Tagged Photos through a Deep Learning Model." Journal of Quality Assurance in Hospitality \& Tourism 21(2):234-43. doi: 10.1080/1528008X.2019.1653243.

Zhang, Zhanpeng, Ping Luo, Chen Change Loy and Xiaoou Tang. 2018. "From Facial Expression Recognition to Interpersonal Relation Prediction." International Journal of Computer Vision 126(5):550-69. doi: 10.1007/s11263-017-1055-1.

Zitouni, M. Sami, Andrzej Sluzek and Harish Bhaskar. 2019. "Visual Analysis of Socio-Cognitive Crowd Behaviors for Surveillance: A Survey and Categorization of Trends and Methods." Engineering Applications of Artificial Intelligence 82:294-312. doi: 10.1016/j.engappai.2019.04.012.

Zuo, Fan, Jingqin Gao, Abdullah Kurkcu, Hong Yang, Kaan Ozbay and Qingyu Ma. 2021. "ReferenceFree Video-to-Real Distance Approximation-Based Urban Social Distancing Analytics Amid Covid-19 Pandemic." Journal of Transport \& Health 21:101032. doi: 10.1016/j.jth.2021.101032. 


\section{Promise Into Practice: Application of Computer Vision in Empirical Research on Social Distancing}

\section{Tables 1-3}

Table 1: Sampling of still frames for different phases of the study

\begin{tabular}{lllllll}
\hline Set & $\begin{array}{l}\text { Video } \\
\text { files }\end{array}$ & $\begin{array}{l}\text { Still } \\
\text { frames }\end{array}$ & Coder 1 & Coder 2 & $\begin{array}{c}\text { Algorithm } \\
\text { version 1 }\end{array}$ & $\begin{array}{l}\text { Algorithm } \\
\text { version 2 }\end{array}$ \\
\hline 1 & 12 & 128 & $\checkmark$ & $\checkmark$ & & \\
2 & 24 & 221 & $\checkmark$ & $\checkmark$ & $\checkmark$ & $\checkmark$ \\
3 & 24 & 201 & $\checkmark$ & & & $\checkmark$ \\
\cline { 1 - 2 } Total & 60 & 550 & & & & \\
\hline
\end{tabular}


Table 2: Human inter-coder reliability measures (Krippendorff's alpha) and 95\% confidence intervals of the number of persons and the number of social distancing violations $(<1.5$ meter) observed by two coders in 221 double-rated still frames (Set 2).

\begin{tabular}{llc}
\hline measure & alpha & $95 \%$ confidence interval \\
\hline persons & 0.98 & {$[0.97-0.98]$} \\
violations & 0.93 & {$[0.90-0.95]$} \\
\hline
\end{tabular}


Table 3: Inter-coder reliability measures (Krippendorff's alpha) and 95\% confidence intervals between one human coder and three versions of the algorithm. The measures are based on 221 still frames (Set 2) and 201 still frames (Set 3).

\begin{tabular}{|rccll|}
\hline Set & alpha & $95 \%$ confidence interval & measure & $\begin{array}{l}\text { algorithm } \\
\text { version }\end{array}$ \\
\hline 2 & 0.85 & {$[0.80-0.90]$} & persons & 1 \\
2 & 0.85 & {$[0.79-0.90]$} & persons & 2 \\
3 & 0.85 & {$[0.71-0.94]$} & persons & 2 \\
\hline 2 & 0.52 & {$[0.24-0.71]$} & violations & 1 \\
2 & 0.85 & {$[0.80-0.89]$} & violations & 2 \\
3 & 0.84 & {$[0.76-0.90]$} & violations & 2 \\
\hline
\end{tabular}




\section{Promise Into Practice: Application of Computer Vision in Empirical Research on Social Distancing}

Figures 1-4

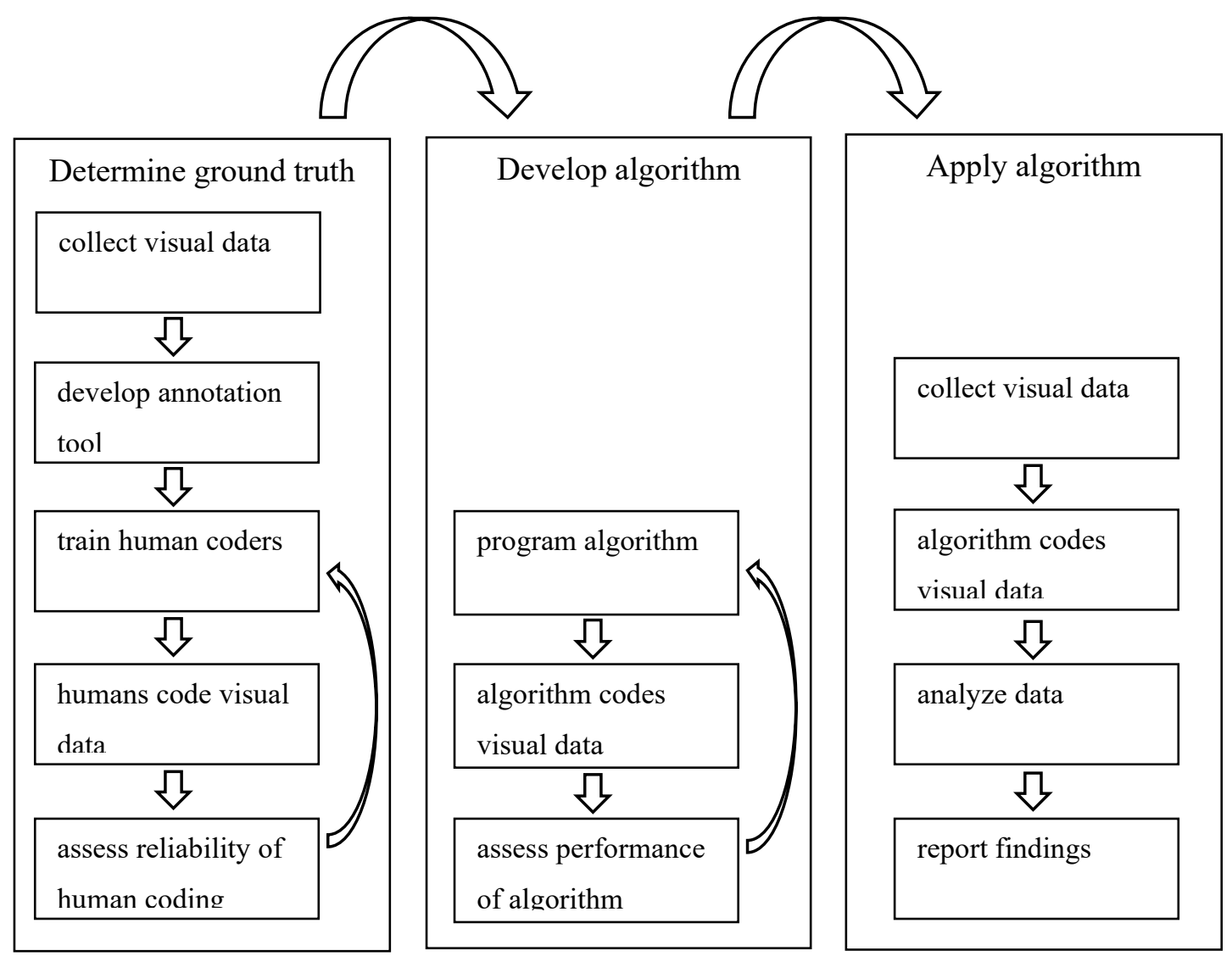

Figure 1: Schematic overview of the process 
[- Annotation Tool

File Help

\begin{tabular}{|c|c|c|c|c|}
\hline Videos & "./Presentation.mp4" & $v$ & Previous video & Next video \\
\hline Frames & SMR_StagedExample.jpg & $\checkmark$ & Previous frame & Next frame \\
\hline
\end{tabular}

\begin{tabular}{l|l|l|l|}
\hline Smaller (s) & Larger (d) Group heads $(\mathrm{g})$ & Delete \\
\hline
\end{tabular}

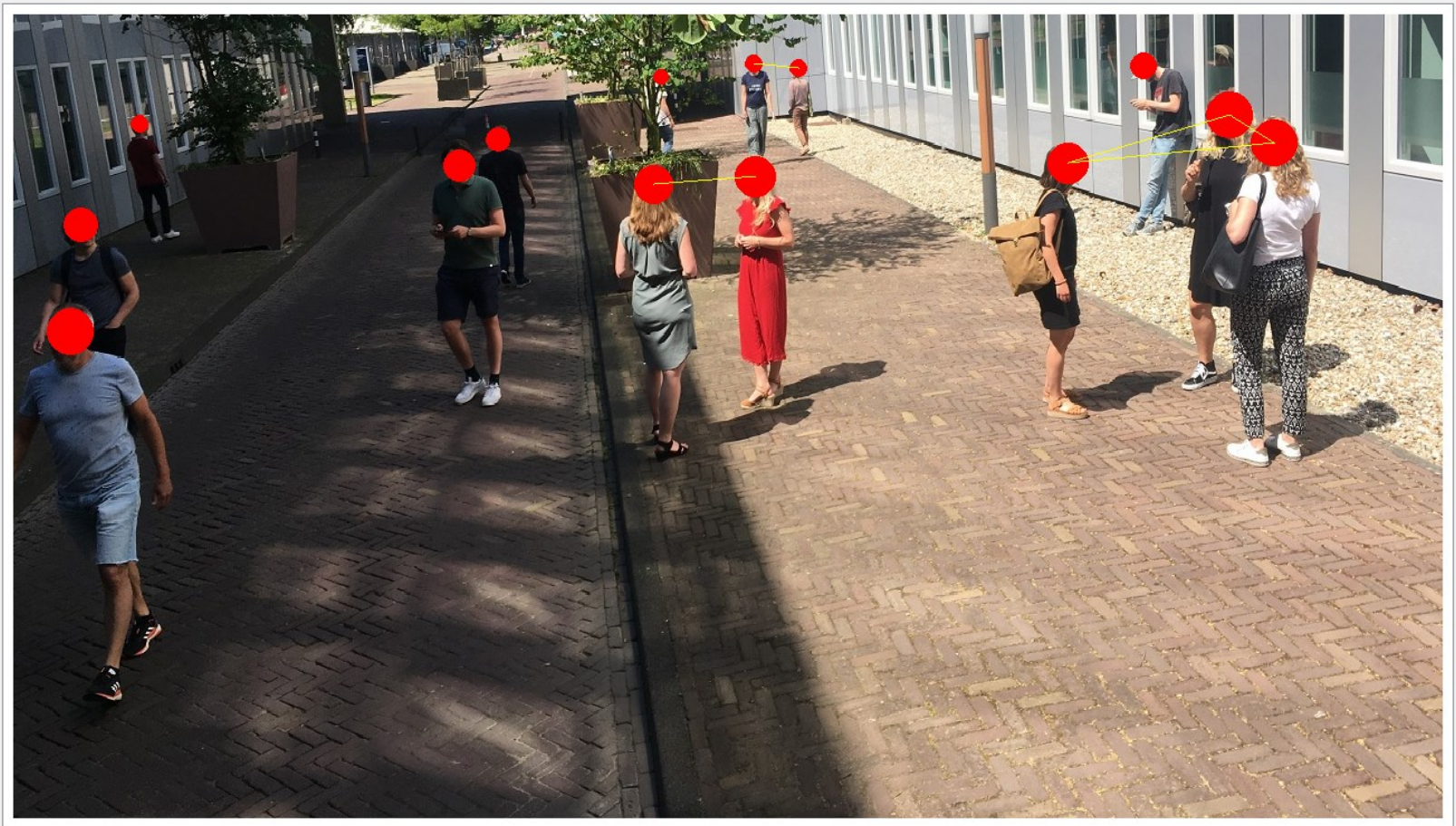

Figure 2. Annotation tool interface. This is a staged scene. All depicted individuals provided written consent for this image to be used for the present purpose. Privacy regulations and data access agreements do not allow sharing recorded CCTV footage. 


\section{A. Perpendicular}

\section{B. In direction of view}
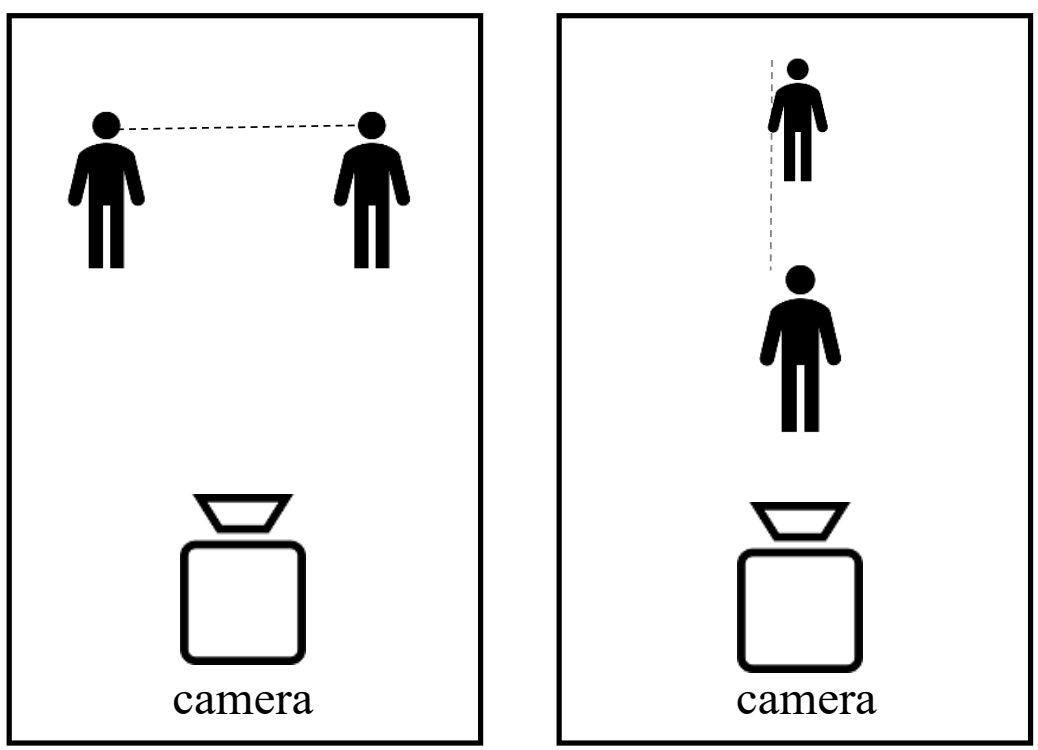

Figure 3: (A) Distance between detected persons perpendicular to the camera view; (B) Distance between detected persons in the direction of the camera view. 


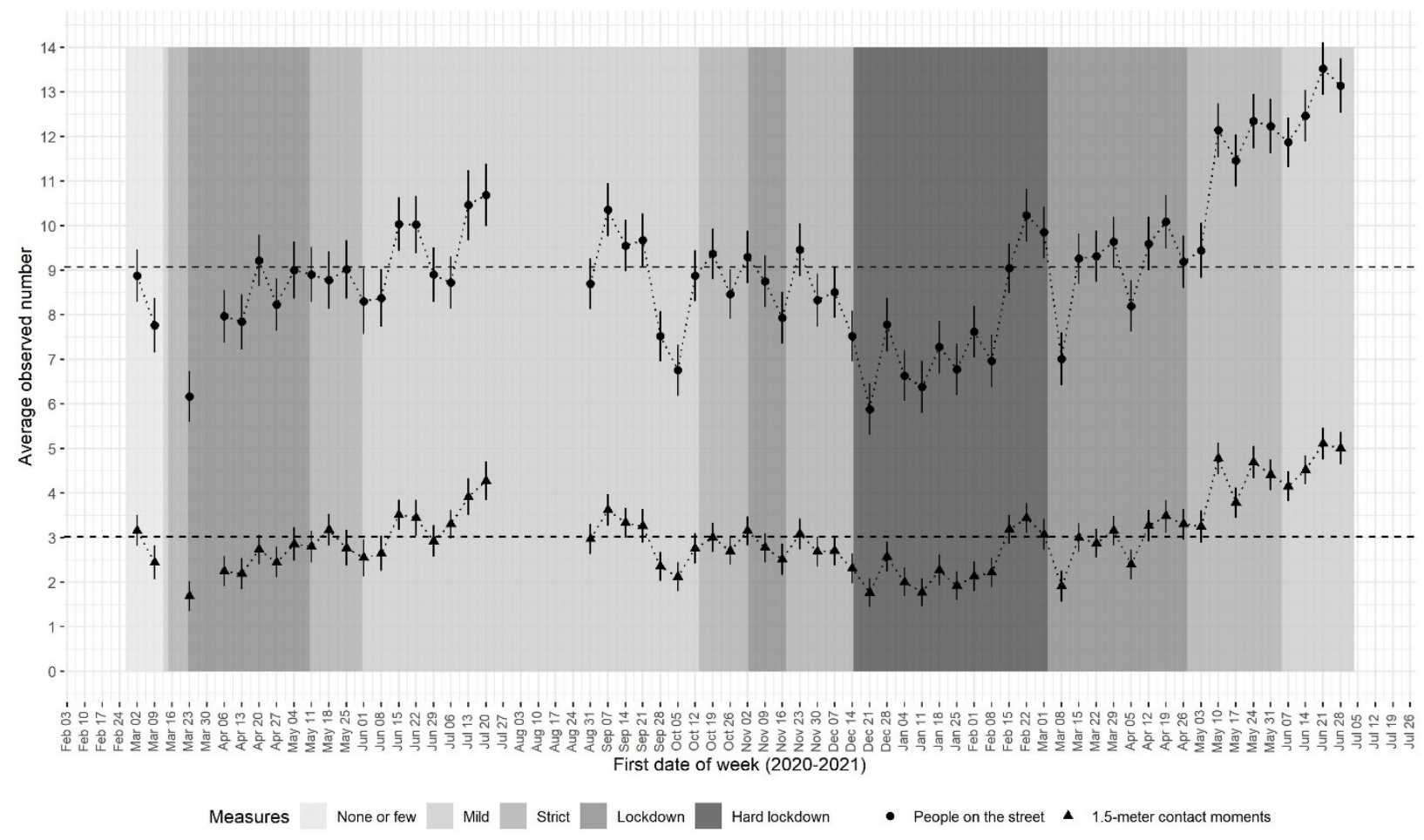

Figure 4: Development of number of persons in the street and number of violations of the $1.5 \mathrm{~m}$ rule during the COVID-19 pandemic. Point estimates (dots) and 95\% confidence intervals (vertical bands). 
Promise Into Practice: Application of Computer Vision in Empirical Research on Social Distancing

\author{
Supplemental Materials 1-4
}




\section{Supplemental Material 1: Coder Instruction Annotation Tool}

Start the annotation tool by double clicking /nscr/src/start_anno.bat.

Use menu "File/Open Video Set" to open VideoData/nscr.txt which contains the list of all videos. You should see the first frame of the first video.

First, select all individuals in the frame. You can do this by right clicking on the head of a person. A red circle will appear. Ideally, the top of the circle is aligned with the top of the head of the person. You can change the size of the selected circles with the "Smaller" and "Larger" button or by pressing the 's' and ' $\mathrm{d}$ ' keys. We start with people closest to the camera and then back, always selecting from left to right. This is to try to ensure you will assign the same person ID (the tool will generate this automatically). We select all individuals visible in the frame.

Second, we will 'group' people who transgress the 1.5-meter guideline. Whenever you see two individuals violating the distance, select the circles (left click on a circle to select it (use ctrl + left to select multiple circles) and use the "Group heads" button or press the ' $\mathrm{g}$ ' key. A yellow line between the individuals will appear. This means that all individuals will have a red dot, but only the individuals who transgress the 1.5-meter guideline will have a yellow line between them. This includes individuals that already seem to be together.

Whenever one individual violates with multiple people, you can add an additional red dot (right click). You cannot use the same dot twice for violations with multiple people.

Whenever the individuals are further away from the camera, it gets harder to make an estimation on whether they keep distance or not. As a rule of thumb, take the individual closest to the camera that is fully visible and measure the number of centimetres (vertically). Whenever the individuals you are observing are one fourth of the measured centimetres or less, stop coding the violations and only select the individual (e.g., individual closest to the camera $=2 \mathrm{~cm}$, select but stop coding when you measure 0,5 cm or less).

Selected circles are removed with the "Delete" button.

\section{Indications of 1.5 meter}

The length of a bike

The distance between two arms

5 big square tiles on the street $=1.5$ meter, 15 small tiles on the street $=1.5$ meter

Sometimes, there are marks on the street indicating 1.5 meter. Keep in mind that whenever the individual you are coding is not aligned with the mark, it will not be 1.5 meter anymore.
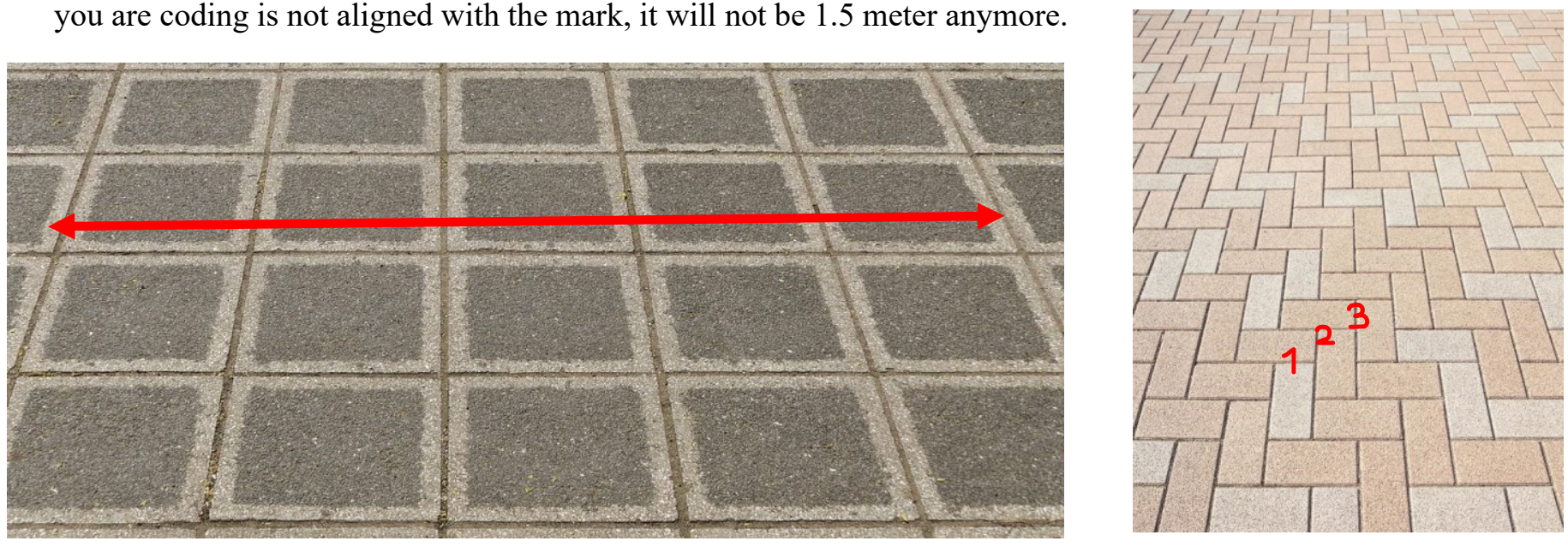


\section{Supplemental Material 2: Visualization agreement human coders}

For additional visual assessment of the agreement between coders, we created scatterplots with the observations of one coder on the horizontal axis and the observations of the other coder on the vertical axis. Panel A in Figure S1 displays the agreement on the number of persons observed per still frame. The size of each dot indicates how many still frames it represents. Dots on the diagonal indicate perfect agreement, while the horizontal and vertical distances from the diagonal represent the level of disagreement. The visualization confirms that the level of agreement is high, as virtually all dots concentrate around the diagonal, with very few outliers.

Panel B in Figure S1 visualizes the coder agreement on the number of social distancing violations of the 1.5-meter distance rule observed in the still frames. Since the observation of a social distancing violation requires the observation of at least two individuals as well as the establishment of the distance between them, it is arguably a more difficult task. Like in Panel A, the dots concentrate around the diagonal, but they appear to display somewhat more diffusion away from the diagonal. Thus, confirming the summary measures in Table 2 , the level of agreement on the violation counts is still high, but slightly lower than the level of agreement on the person counts.

A

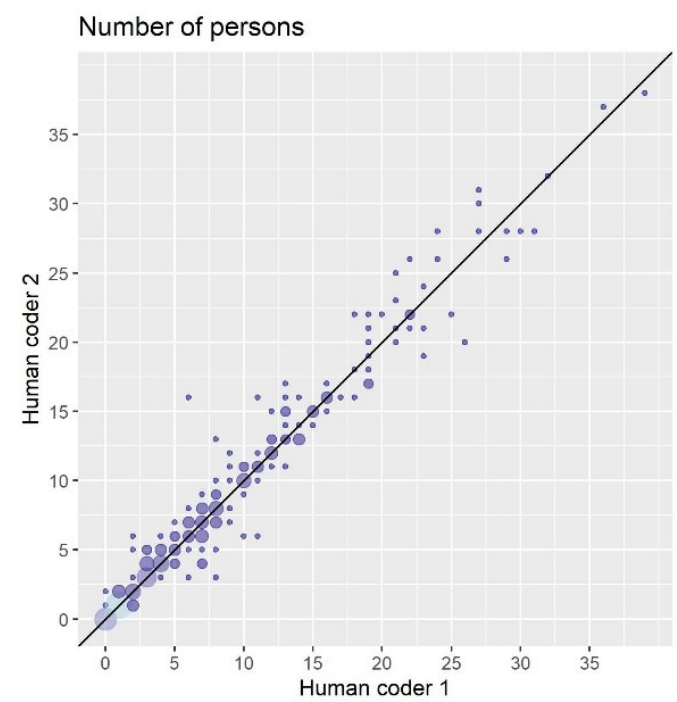

B

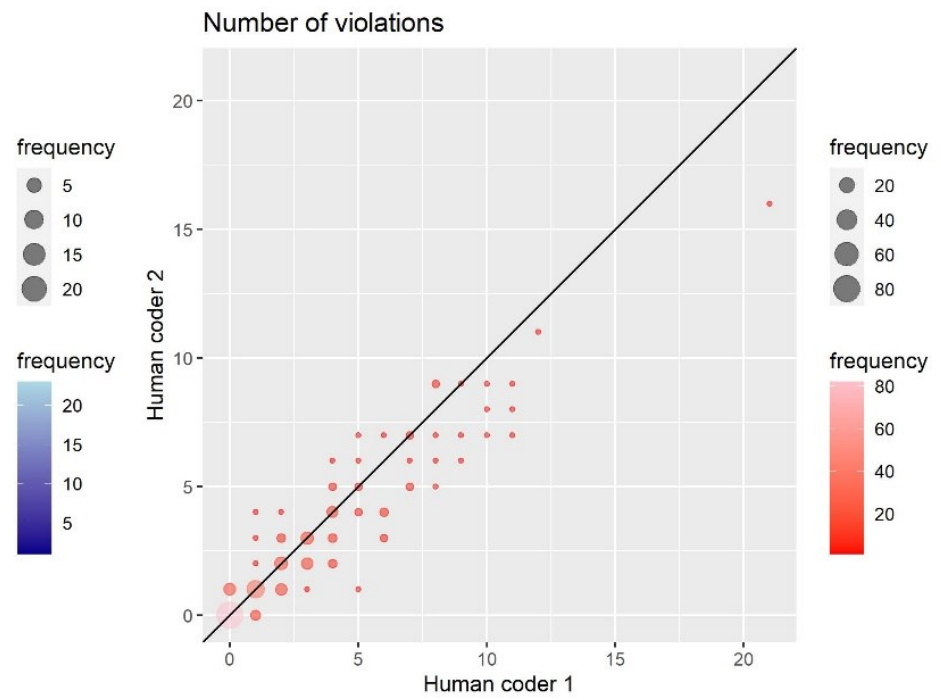

Figure S1: (A) Scatter plot of person counts by human coders 1 and 2 (B) Scatter plot of violation counts by human coders 1 and 2. Observed in in 221 still frames (Set 2). 


\section{Supplemental Material 3: Visualization agreement human coder and algorithm}

The disagreement between the ratings of the first version of the algorithm and the human coder is visualized in Figure S2, with the observations of the human coder on the horizontal axis and the observations of the algorithm on the vertical axis. Although in panel A the large majority of the dots suggest a satisfactory performance — as indicated by their location on or near the diagonal — there appears to be a slight tendency of the algorithm to underestimate the number of persons in the still frame, as indicated by the dots that are located below the diagonal. In panel B most observations lie below the diagonal, which suggests that the weak performance of the algorithm is a function of the algorithm being more conservative in assessing distance violations than the human coder.

The relative agreement between the second version of the algorithm and the human coder is visualized in Figure S3. Although the agreement is not perfect, panel B in Figure S3 no longer shows the downward bias of the algorithm that was visible in Figure S2.

A

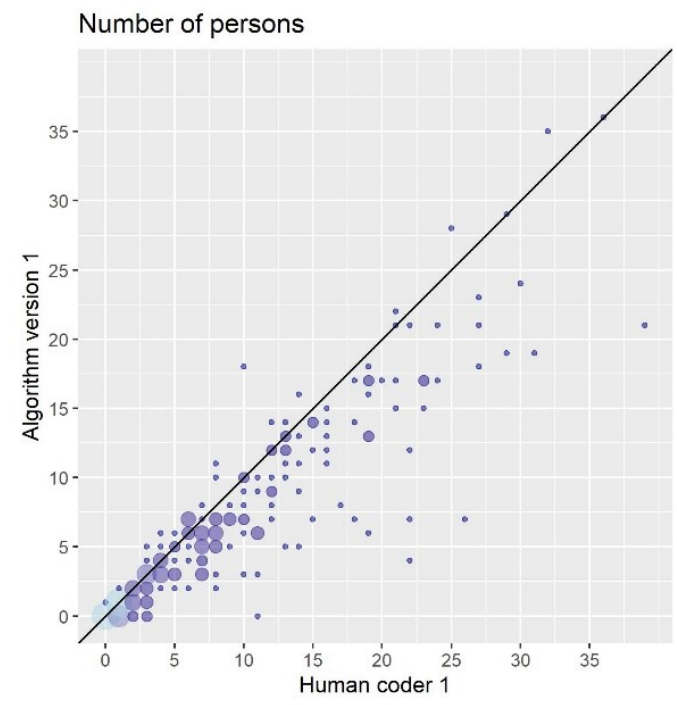

B

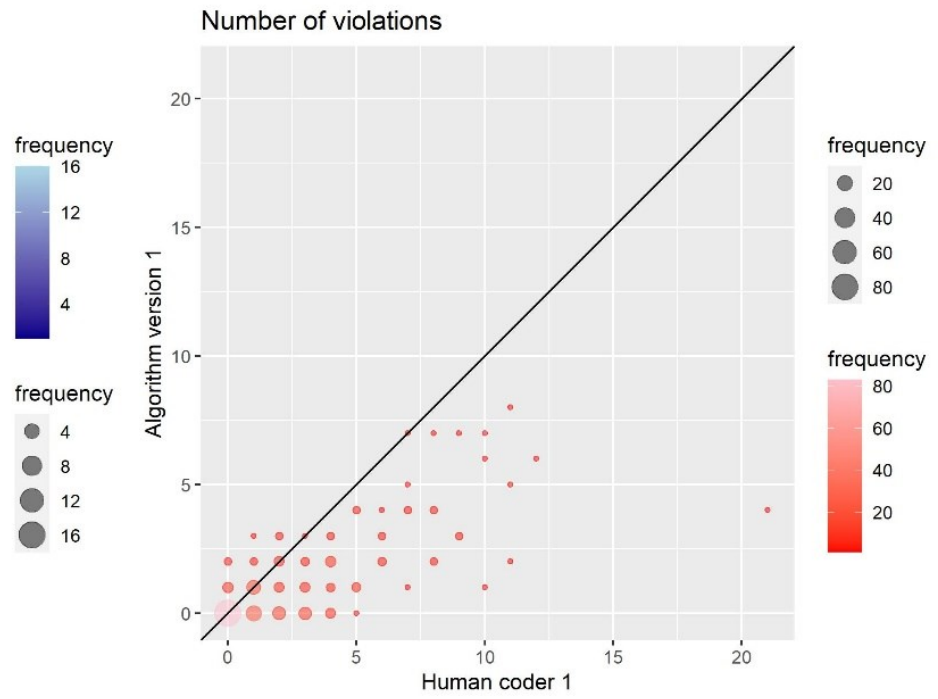

Figure S2: (A) Scatter plot of person counts by human coder 1 and the first version of the algorithm (B) Scatter plot of violation counts by human coder 1 and the first version of the algorithm. Observed in 221 still frames (Set 1). 
A

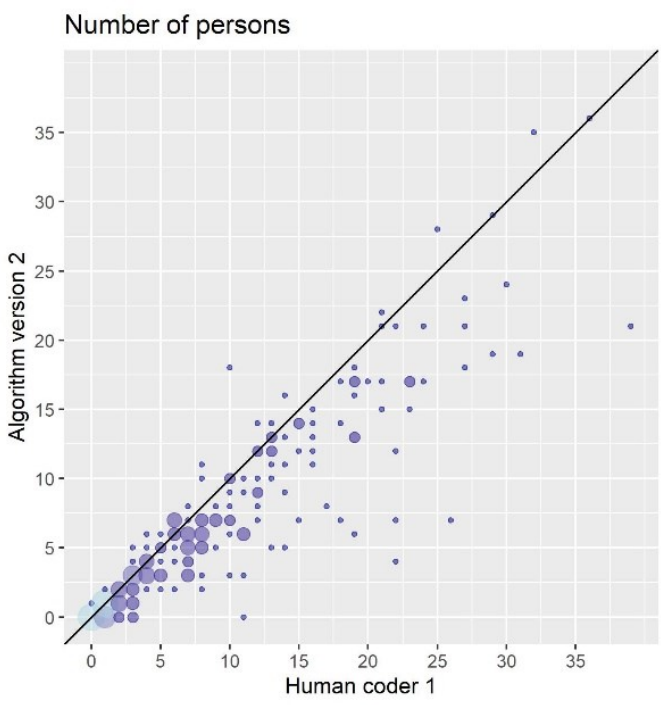

B

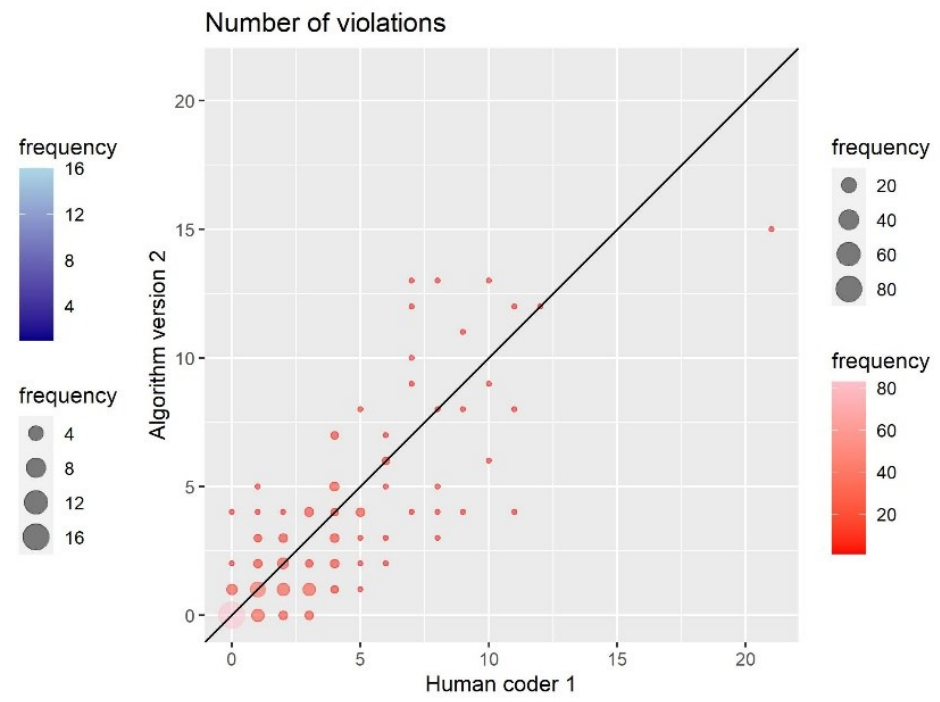

Figure S3: (A) Scatter plot of person counts by human coder 1 and the second version of the algorithm (B) Scatter plot of violation counts by human coder 1 and the second version of the algorithm. Observed in in 221 still frames (Set 2). 


\section{Supplemental Material 4: Multiple Imputation}

Figure S4 displays the distribution of proportions of missing values across days, cameras and hours of the day, ordered by proportion of missing values. The relation with hour of the day is systematic, with every later hour having a larger proportion of missing values. Some cameras have large proportions of missing data. The 8 cameras with more than 60 percent missing values were not used in the data analysis.

\section{Multiple imputation procedure evaluation}

Before implementing a multiple imputation procedure, we first explored the feasibility of the procedure for our data. To that end, we investigated what parameters are most likely to be a optimal, and evaluated the quality of the resulting imputation. The quality of multiple imputation was evaluated by simulating the generation of missing values and the multiple imputation procedure on a subset of the data that contained no missing values (see, van Buuren 2018:51-53). The evaluation was performed in January 2021 and is therefore limited to data on observations made during 2020.

In the data analysis, our multiple imputation setup involved (a) using camera, hour of the day and date (all nominal level) as predictive variables for imputations of both numbers of persons and numbers of violations, (b) using 100 imputations (i.e. alternative versions of the dataset with imputed missing values, and (c) using "predictive mean matching" as the prefered method of imputation. The $\mathrm{R}$ code conducting the multiple imputation procedure and the use of the imputed datasets for data analysis is available at https://osf.io/yw67g. 

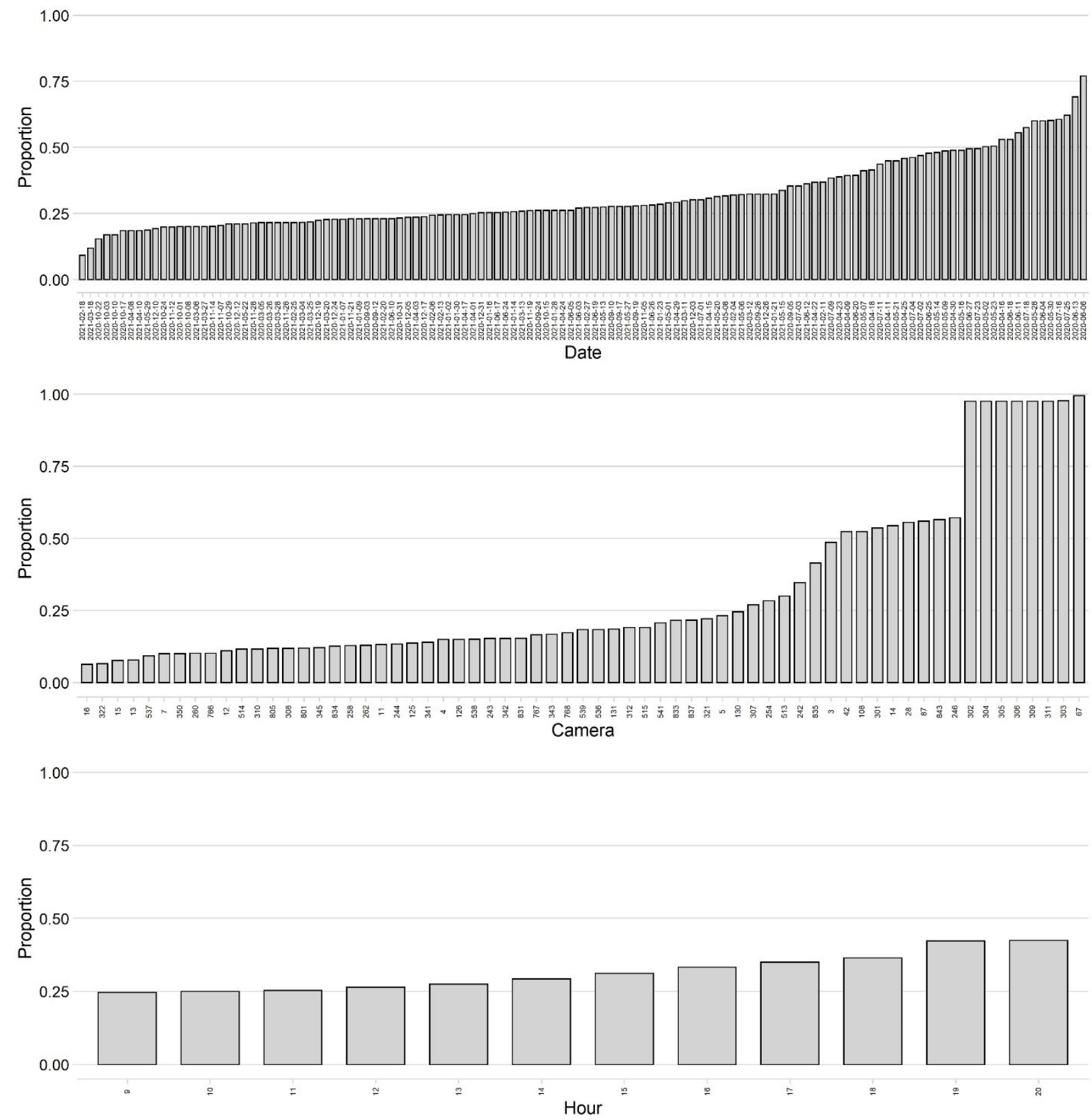

Figure S4: Proportion of missing observations per date, camera and hour (ordered by proportion missing observation) 